\title{
Fauna of longicorn beetles (Coleoptera: Cerambycidae) of Mordovia
}

\section{Фауна жуков-усачей (Coleoptera: Cerambycidae) Мордовии}

\author{
A.B. Ruchin ${ }^{1}$, L.V. Egorov ${ }^{1,2}$ \\ A.Б. Ручин ${ }^{1}, \Lambda . B$. Егоров $^{1,2}$ \\ ${ }^{1}$ Joint Directorate of the Mordovia State Nature Reserve and National Park «Smolny», Dachny per., 4, Saransk 430011, Russia.
${ }^{1}$ ФГБУ «Заповедная Мордовия», Дачный пер., 4, г. Саранск 430011, Россия. E-mail: sasha_ruchin@rambler.ru
${ }^{2}$ State Nature Reserve «Prisursky», Lesnoi, 9, Cheboksary 428034, Russia. E-mail: platyscelis@mail.ru
${ }^{2}$ ФГБУ «Государственный заповедник «Присурский», пос. Лесной, 9, г. Чебоксары 428034, Россия.
}

KEY WORDS: Coleoptera, Cerambycidae, Russia, Mordovia, fauna.

КЛЮЧЕВЫЕ СЛОВА: Coleoptera, Cerambycidae, Россия, Мордовия, фауна.

ABSTRACT. This paper presents an overview of the Cerambycidae fauna in Mordovia, based on available literature data and our own materials, collected in 2002-2017. It provides information on the distribution in Mordovia, and some biological features for 106 species from 67 genera. From the list of fauna are excluded Rhagium bifasciatum, Brachyta variabilis, Stenurella jaegeri, as their habitation in the region is doubtful. Eight species are indicated for the republic for the first time. There were found extremely rare for Russia Leptura aurulenta Fabricius, 1793 and Phymatodes abietinus Plavilstshikov et Lurie, 1960.

РЕЗЮМЕ. Представлен обзор фауны Cerambycidae Мордовии, основанный на имеющихся литературных данных и собственных материалах, собранных в 2002-2017 гг. Для 106 видов из 67 родов приведены сведения о распространении в Мордовии и некоторых биологических особенностях. Из списка фауны исключены Rhagium bifasciatum, Brachyta variabilis, Stenurella jaegeri, обитание которых в пределах региона сомнительно. Впервые для республики указывается 8 видов. Обнаружены очень редкие для России Leptura aurulenta Fabricius, 1793 и Phymatodes abietinus Plavilstshikov et Lurie, 1960.

\section{Introduction}

Longicorn beetles (Cerambycidae), is one of the most studied and economically important groups of Coleoptera in the fauna of Russia. At the moment, reliable lists of species of the family have been published for a number of regions of the central part of European part of Russia. Specificly, fauna of Cerambycids is fairly well studied for Moscow [Danilevsky, 2005], Ulyanovsk [Isajev et al., 2004; Isajev, 2007],
Tula [Bolshakov, Dorofeev, 2004], Yaroslavl [Vlasov, 1999], Kaluga [Aleksanov, Alekseev, 2003], Samara [Isajev, 2007] regions, Udmurt [Dedyukhin, 2007] and Chuvash [Egorov, 2005, 2006] Republics. The first survey work on the fauna of Longicorns in Mordovia Republic was published by us [Ruchin, 2008a]. There were indicated 55 species from 37 genera, found in the region. At the same time, Ergates faber (Linnaeus, 1760), Anastrangalia dubia (Scopoli, 1763), Stictoleptura fulva (DeGeer, 1775) and Aromia moschata ambrosiaca (Steven, 1809) were excluded from the list, as their indication for the republic [Redikortsev, 1938; Plavilshchikov, 1964; Bondarenko, 1964; Mozolevskaya et al., 1971; Timraleev, 1999; Ruchin et al., 2007; Timraleev et al., 2007] were clearly unreliable. In subsequent years, ongoing research was carried out on the territory of Mordovia Reserve, Smolny National Park and their surroundings [Ruchin, Grishutkin, 2008; Ruchin, Kurmaeva, 2009, 2010; Egorov et al., 2010, 2015, 2016, 2017; Orlov et al., 2011; Egorov, Ruchin, 2012, 2013a, 2014; Ruchin, 2011, 2015a, b; Ruchin et al., 2014, 2017] and in the city of Saransk [Ruchin, 2009; Semishin, 2009].

\section{Material and methods}

The Republic of Mordovia is located in the center of the East European Plain in the interfluve of the rivers Moksha and Sura, mainly in the forest-steppe landscape zone, although in its northwestern part there is an array of forests of the southern taiga, and in the south there are still preserved some steppe areas (in many regions there is a significant number of steppe sites) [Yamashkin, 1998]. The variety of natural conditions determines the fauna features of the republic.

Material for this paper was collected during seasons in 2002-2017 (most intensively since 2007) using the

How to cite this article: Ruchin A.B., Egorov L.V. 2018. Fauna of longicorn beetles (Coleoptera: Cerambycidae) of Mordovia // Russian Entomol. J. Vol.27. No.2. P.161-177. doi: 10.15298/rusentj.27.2.07 
generally accepted entomological methods of field research [Fasulati, 1971]. In total, more than 4,000 specimens were examined during the study.

There are given references to the literature on the species from the territory of Mordovia (in case there is a reliable indication of the point of discovery it is mentioned ether), new collection sites (previously unpublished information), collection date, number of collected specimens, surname of the collector, notes (if any) for each species in the annotated list below.

When the collector is not mentioned it means that material was collected by the first author of the paper. The original information on the biology of anthophilous longicorns, species habitats description, and certain observations in nature are given separately. The names of new species for the Republic of Mordovia are marked with an asterisk (*). The sign "_" indicates the species, which, for various reasons (see comments), we exclude from the list of Mordovia longicorns. The sign "??" indicates that the findings of this species require confirmation by modern materials.

The nomenclature of Cerambycidae is adopted according to the publications of M.L. Danilevsky [2014, 2017]. To clarify the general distribution of species, in addition to the above mentioned, M.L. Danilevsky, the information from the Catalogue of Palaearctic Coleoptera [2010] was used. All the collection material is stored in the funds of Zoological Museum of Moscow State University (Moscow), Zoological Institute of Russian Academy of Sciences (St. Petersburg), Museum of Mordovia Reserve (Pushta), Biological Museum of Mordovia State University (Saransk) and in the personal collections of authors.

The abbreviations used are: MSNR - Mordovia State Nature Reserve, NPS — National Park "Smolny", ex., - specimen.

\section{Species list}

\section{Subfamilia Prioninae \\ Prionus coriarius (Linnaeus, 1758)}

MATERIAL. Temnikov Distr.: MSNR, 25 km E Temnikov, 7.VIII.2017, 2 ex., Bolshakov L.V.; 15 km N Temnikov, 27.VII.6.VIII.2017, 9-27.IX.2017, 2 ex., Egorov L.V., Semishin G.B.; Zubova Polyana Distr.: Kargashino, 29.VII.2009, 1 ex.; Shiringushi, 31.VII.2009, 1 ex.; Krasnoslobodsk Distr.: Staroe Sindrovo, 1.VIII.2008, 1 ex.; Ichalki Distr.: NPS, 9 km NE Ichalki, 31.VII.2017, 1 ex., Semishin G.B.; Lyambir Distr.: Penzyatka, VI-VIII.2014, 1 ex.

DISTRIBUTION in Mordovia. Temnikov Distr. [Plavilshchikov, 1964; Mozolevskaya et al., 1971; Ruchin, Kurmaeva, 2009; Egorov, Ruchin, 2012, 2013a; Egorov et al., 2016, 2017]; Krasnoslobodsk Distr. [Ruchin, Kurmaeva, 2009]; Ichalki Distr. [Ruchin, 2008a; Ruchin, Grishutkin, 2008]; Dubenki Distr. [Ruchin, 2008a]; Kochkurovo Distr. [Semishin, 2009]; Bolshie Berezniki Distr. [Timraleev, 1999; Ruchin, 2008a]. Saransk Town [Ruchin, 2008a, 2009]. Zubova Polyana Distr. [this paper].

BIOLOGY in Mordovia. Found in pine forests, mixed forests, oak groves. Flies to the light. Flight of the imago lasts from 1st decade of July to 3rd decade of September.

\section{Subfamilia Lepturinae \\ Oxymirus cursor (Linnaeus, 1758)}

DISTRIBUTION in Mordovia. Temnikov Distr. [Feoktistov, 2011; Ruchin, Kurmaeva, 2009; Egorov et al., 2017].

BIOLOGY in Mordovia. Recorded in pine-bilberry-green moss forests, ripe pine forests with spruce, birch. Flight of the imago lasts from 2nd decade of May to 2nd decade of July.

Rhamnusium bicolor (Schrank, 1781)

In Mordovia there is a subspecies $R$. bicolor constans Danilevsky, 2012.

DISTRIBUTION in Mordovia. Temnikov Distr. [Egorov, Ruchin, 2013a].

BIOLOGY in Mordovia. The only find on the trunk of the birch. Occurs in deciduous forests, often in floodplains. It is associated with various deciduous tree species [Shapovalov, 2012].

\section{- Rhagium bifasciatum Fabricius, 1775}

REMARK. Indicated for Mordovia Reserve [Mozolevskaya et al., 1971). This, most likely, is about the Rhagium sycophanta. Within the European part of Russia, $R h$. bifasciatum is found in the Caucasus [Danilevsky, Miroshnikov, 1985; Danilevsky, 2014].

\section{Rhagium inquisitor (Linnaeus, 1758)}

MATERIAL. Temnikov Distr.: MSNR, $16 \mathrm{~km} \mathrm{~N}$ Temnikov, 13.V.2017, 1 ex., Egorov L.V.; 15 km N Temnikov, 14.V.2017, 12.V.-1.VI.2017, 2.VI.2017, 1-15.VI.2017, 20 ex., Egorov L.V., Semishin G.B.; Ichalki Distr.: NPS, Kemlyanskoe forestry, $4 \mathrm{~km}$ NNE Ichalki, 18.IV.2009, 12 ex.; NPS, Lvovskoe forestry, $15 \mathrm{~km}$ NNE Ichalki, 18.IV.2009, 2 ex.; same label, 23.V.2017, 19.VI.3.VII.2017, 2 ex., Semishin G.B.

DISTRIBUTION in Mordovia. Temnikov Distr. [Plavilshchikov, 1964; Ruchin, Kurmaeva, 2009; Egorov, Ruchin, 2012, 2013a, 2014; Egorov et al., 2015, 2016, 2017]; Zubova Polyana Distr. [Ruchin, Kurmaeva, 2009]; Bolshie Berezniki Distr. [Timraleev, 1999]. Ichalki Distr. [this paper].

BIOLOGY in Mordovia. It inhabits mixed and coniferous forests. In Mordovia Reserve larvae and pupae were often found under the bark of pine trees [Redikortsev, 1938]. Chitinous remains of larvae were found in the spring litter of Ursus arctos Linnaeus, I758. In Smolny NP larvae were also found under the bark of fallen pine trees and pine logs. Imago flight lasts from 3 rd decade of April to 2 nd decade of July.

\section{Rhagium mordax (DeGeer, 1775)}

MATERIAL. Temnikov Distr.: Veselyj, 6.VI.2015, 1 ex.; Temnikov Distr.: MSNR, 22 km N Temnikov, 20.V.2017, 1 ex.; MSNR, 18 km N Temnikov, 20.V.2017, 1 ex.; MSNR, 15 km N Temnikov, 13-27.VII.2017, 1 ex., Egorov L.V., Semishin G.B.; Lyambir Distr.: Ekaterinovka, 9.V.2008, 1 ex.; Torbeevo Distr.: Vindrej, 6.VI.2008, 1 ex.; Ichalki Distr.: NPS, 15 km NNE Ichalki, 31.V.2009, 1 ex., Artaev O.N.; Elniki Distr.: Novye Pichingushi, IV-VI.2014, 1 ex.; Ardatov Distr.: Svetotekhnika, 18.V.2008, 1 ex.; Oktyabrsky, 19.V.2008, 1 ex.; 4,5 km SW Oktyabrsky, 19.V.2008, 1 ex.; Chamzinka Distr.: Bolshoe Maresevo, 13.VI.2008, 1 ex.; Dubenki Distr.: 8 km SE Engalychevo, 1.VII.2009, 1 ex.

DISTRIBUTION in Mordovia. Tengushevo Distr. [Ruchin, Kurmaeva, 2009]; Temnikov Distr. [Ruchin, Kurmaeva, 2009; Feoktistov, 2011; Egorov, Ruchin, 2012, 2013a, 2014; Egorov et al., 2015, 2016, 2017]; Zubova Polyana Distr. [Ruchin, 2008a; Ruchin, Kurmaeva, 2009]; Torbeevo Distr. [Ruchin, Kurmaeva, 2009]; Bolshoe Ignatovo Distr. [Ruchin, 2008a]; Ichalki Distr. [Ruchin, 2008a; Ruchin, Grishutkin, 2008]; Ruzaevka Distr. [Ruchin, 2008a]; Bolshie Berezniki Distr. [Timraleev, 1999; Ruchin, Grishutkin, 2008]; 
Kochkurovo Distr. [Semishin, 2009]. Saransk Town [Ruchin, 2008a, 2009; Semishin, 2009]. Lyambir Distr., Elniki Distr., Ardatov Distr., Chamzinka Distr., Dubenki Distr. [all this paper].

BIOLOGY in Mordovia. It is quite common in deciduous, mixed, pine forests, birch plantations. Found on the inflorescences of Padus avium Mill., Sorbus aucuparia L., Filipendula ulmaria (L.) Maxim., Rubus idaeus L., Cerasus vulgaris Mill., and also while mowing on Salix sp. Imago flight lasts from 1st decade of May to 2 nd decade of July.

\section{Rhagium sycophanta (Schrank, 1781)}

MATERIAL. Ruzaevka Distr.: Levzhensky, 9.VI.2009, 1 ex.; Bolshie Berezniki Distr.: 9 km S Simkino, 20.VI.2008, 1 ex., Lobachev E.A.; Kochkurovo Distr.: Starye Turdaki, VI.2008, 1 ex.

DISTRIBUTION in Mordovia. Temnikov Distr. [Ruchin, Kurmaeva, 2009; Feoktistov, 2011; Egorov, Ruchin, 2013a; Egorov et al., 2017]. Krasnoslobodsk Distr. [Ruchin, Kurmaeva, 2009]; Kovylkino Distr. [Ruchin, 2008a]; Bolshie Berezniki Distr. [Timraleev, 1999]; Bolshie Berezniki Distr. [Ruchin, 2008a]. Ruzaevka Distr., Kochkurovo Distr. [all this paper].

BIOLOGY in Mordovia. Occurs in deciduous forests, especially in oak groves. Imago flight lasts from 1st decade of May to 2nd decade of July.

Stenocorus meridianus (Linnaeus, 1758)

MATERIAL. Ichalki Distr.: NPS, Kemlyanskoe forestry, $7 \mathrm{~km}$ N Ichalki, 27.VII.2017, 1 ex.

DISTRIBUTION in Mordovia. Temnikov Distr. [Egorov et al., 2010; Ruchin et al., 2010, 2017; Egorov, Ruchin, 2014]; Staroe Shajgovo Distr. [Ruchin et al., 2010]; Ardatov Distr. [Ruchin et al., 2010]; Chamzinka Distr. [Ruchin, 2008a; Ruchin et al., 2010, 2017]; Ruzaevka Distr. [Ruchin et al., 2010]; Bolshie Berezniki Distr. [Ruchin et al., 2010]; Kochkurovo Distr. [Semishin, 2009]. Saransk Town [Ruchin, 2009; Ruchin et al., 2010, 2014]. Ichalki Distr. [this paper].

BIOLOGY in Mordovia. Habitats are deciduous and mixed forests. Found on the inflorescences of Aegopodium podagraria L., Angelica sylvestris L., Anthriscus sylvestris (L.) Hoffm., and also on the leaf of Padus avium Mill. Imago flight lasts from 3rd decade of May to 3rd decade of July.

\section{Stenocorus quercus (Götz, 1783)}

DISTRIBUTION in Mordovia. Kochkurovo Distr. [Ruchin, 2008b; Ruchin, Egorov, 2015].

BIOLOGY in Mordovia. The only location in the republic is the edge of deciduous forest of secondary origin. A rare species in the northern part of the range. Larvae develop in dead rotting roots of oak and maple deep under the ground, usually far from the trunk [Danilevsky, 2014].

Pachyta quadrimaculata (Linnaeus, 1758)

MATERIAL. Tengushevo Distr.: $6 \mathrm{~km}$ W Barashevo, 18.VII.2015, 4 ex.; Elniki Distr.: Novye Shaly, 19.VII.2015, 4 ex.; Malye Mordovskie Poshaty, 22.VII.2017, 1 ex.; Staroe Shajgovo Distr.: Lesnichestvo, 17.VII.2009, 1 ex.; Ichalki Distr.: NPS, Lvovskoe forestry, $15 \mathrm{~km}$ NNE Ichalki, 30.VI.2008, 5.VII.2008, $2 \mathrm{ex}$.

DISTRIBUTION in Mordovia. Tengushevo Distr. [Ruchin et al., 2015, 2016]. Temnikov Distr. [Plavilshchikov, 1964; Orlov et al., 2011; Egorov, Ruchin, 2012, 2013a, 2014; Egorov et al., 2017; Ruchin et al., 2010, 2014, 2015, 2016, 2017]; Elniki Distr. [Ruchin et al., 2016]; Staroe Shajgovo Distr. [Ruchin et al., 2010]; Ichalki Distr. [Ruchin, 2008a; Ruchin, Grishutkin, 2008; Ruchin et al., 2010].
BIOLOGY in Mordovia. It is common in deciduous and mixed forests, where it occurs along fringes, glades, roadsids. It can often be seen on the inflorescences of Angelica sylvestris L., Aegopodium podagraria L., Leucanthemum vulgare Lam., Filipendula ulmaria (L.) Maxim., Pimpinella saxifra$g a \mathrm{~L}$. Imago flight lasts from 1st decade of June to 1st decade of August.

Evodinellus borealis (Gyllenhal, 1827)

DISTRIBUTION in Mordovia. Temnikov Distr. [Egorov, Ruchin, 2013a; Ruchin, Egorov, 2015; Ruchin et al., 2016, 2017].

BIOLOGY in Mordovia. In Mordovia it is common only in Mordovia reserve in mixed forests, in floodplain mixed forests with oak, birch, alder and aspen, pine. Single-marked on Leucanthemum vulgare Lam.

\section{Brachyta interrogationis (Linnaeus, 1758)}

In Mordovia there is a subspecies $B$. interrogationis russica (Herbst, 1784).

MATERIAL. Ichalki Distr.: NPS, Lvovskoe forestry, $15 \mathrm{~km}$ NNE Ichalki, 27.VI.2008, 1 ex.; Bolshoe Ignatovo Distr.: NPS, Aleksandrovskoe forestry, Lesnoy, 31.V.2009, 1 ex., Artaev O.N.; Lyambir Distr.: Ekaterinovka, 29.V.2008, 2.VI.2009, 18 ex.; Insar Distr.: Kochetovka, 4.VI.2016, 1 ex.; Ruzaevka Distr.: Levzhensky, 9.VI.2009, 28 ex.; 3 km N Streletskaya Sloboda, 20.VI.2009, 3 ex.

DISTRIBUTION in Mordovia. Temnikov Distr. [Egorov, Ruchin, 2013a; Egorov et al., 2015, 2017]; Ichalki Distr. [Ruchin, 2008a; Ruchin, Grishutkin, 2008]; Bolshie Berezniki Distr. [Timraleev, 1999]; Kochkurovo Distr. [Semishin, 2009]. Saransk Town [Ruchin, 2008a, 2009; Semishin, 2009]. Bolshoe Ignatovo Distr., Lyambir Distr., Insar Distr., Ruzaevka Distr. [all this paper].

BIOLOGY in Mordovia. The species is confined to the fringes of deciduous and mixed forests, where it adheres to forest glades with meadow motley grass. It was observed on inflorescences of Aegopodium podagraria, Polygonum bistorta L., Rosa sp., Campanula patula L., Euphorbia virgata Waldst. et Kit., Anthriscus sylvestris (L.) Hoffm., Trifolium montanum L., Geranium pratense L., Padus avium Mill., Ranunculus acris L., different species of Taraxacum. Imago flight lasts from 2nd decade of May to 2nd decade of July.

\section{- Brachyta variabilis (Gebler, 1817)}

REMARK. It is indicated as "Evodinus variabilis Gebl." for Mordovia reserve [Mozolevskaya et al., 1971]. The species is distributed from the Far East to the Urals (there are no reliable findings beyond the Volga) [Danilevsky, 2014]. Most likely, the indication is based on an erroneous definition.

\section{Carilia virginea (Linnaeus, 1758)}

MATERIAL. Ichalki Distr.: NPS, Barakhmanovskoe forestry, 13 km NNE Ichalki, 24.V.2008, 1 ex.; 13.VI.2009, 1 ex.

DISTRIBUTION in Mordovia. Temnikov Distr. [Plavilshchikov, 1964; Mozolevskaya et al., 1971; Ruchin, Kurmaeva, 2009; Egorov, Ruchin, 2013a; Egorov et al., 2017]; Zubova Polyana Distr. [Ruchin, Kurmaeva, 2009]. Ichalki Distr. [this paper].

BIOLOGY in Mordovia. In mixed and pine forests. It was noted on the inflorescences of Sorbus aucuparia L. and Plantago media L. Imago flight lasts from 1st decade of May to 2nd decade of June.

Euracmaeops angusticollis (Gebler, 1833)

DISTRIBUTION in Mordovia. Temnikov Distr. [Egorov, Ruchin, 2013a; Egorov et al., 2015, 2016]. 
BIOLOGY in Mordovia. Within the republic it is regularly found only in Mordovia reserve in mixed forests, spruce groves. There is a single finding on the inflorescence of Sorbus aucuparia L. Imago flight lasts from 1st decade of May to 2nd decade of June.

Euracmaeops marginatus (Fabricius, 1781)

DISTRIBUTION in Mordovia. Temnikov Distr. [Egorov, Ruchin, 2013a, 2014; Egorov et al., 2015, 2017].

BIOLOGY in Mordovia. Within the republic is commonly found only in Mordovia nature reserve. It inhabits old high-bonitat pine forests, the larva develops under the bark of dead trees. We have observed it in ripe pine forests with an admixture of spruce, birch, aspen. Imago flight lasts from 2nd decade of May to 1st decade of July.

Euracmaeops septentrionis (Fabricius, 1793)

DISTRIBUTION in Mordovia. Temnikov Distr. [Egorov et al., 2016].

BIOLOGY in Mordovia. A single finding in Mordovia reserve. Biotope - ripe spruce forest with pine, birch. Judging by the spread of the species in the European part of Russia [Danilevsky, 2014] our find is one of the most southerly in this region.

Gnathacmaeops pratensis (Laicharting, 1784)

MATERIAL. Temnikov Distr.: MSNR, 18 km N Temnikov, 4.VII.2017, 2 ex

DISTRIBUTION in Mordovia. Temnikov Distr. [Plavilshchikov, 1964; Egorov et al., 2015, 2016, 2017; Ruchin et al., 2017].

BIOLOGY in Mordovia. Within the republic it is regularly found only in MSNR. Habitats are mixed and pine forests. Imago flight lasts from 2nd decade of May to 1st decade of July.

\section{Dinoptera collaris (Linnaeus, 1758)}

MATERIAL. Tengushevo Distr.: $6 \mathrm{~km} \mathrm{~W}$ Barashevo, 18.VII.2015, 1 ex.; Temnikov Distr.: Babeevo, 8.VI.2013, 1 ex.; Temnikov Distr.: MSNR, 15 km N Temnikov, 14.VI.2017, 1 ex., Semishin G.B.; 2.VII.2017, 1 ex.; 31 km NE Temnikov, 13.VII.2017, 2 ex.; Ichalki Distr.: Khanineevka, 12.VI.2009, 2 ex.; NPS, Lvovskoe forestry, $15 \mathrm{~km}$ NNE Ichalki, 17.VI.2008, 1 ex.; NPS, Barakhmanovskoe forestry, $13 \mathrm{~km}$ NNE Ichalki, 13.VI.2009, 1 ex.; Chamzinka Distr.: Komsomolskij, VI.2008, VII.2009, 2 ex., Ryzhov M.K.; Bolshoe Maresevo, 13.VI.2008, 2 ex.; Lyambir Distr.: Atemar, 24.VI.2017, 1 ex.; Ruzaevka Distr.: Levzhensky, 9.VI.2009, 8 ex.; 3 km N Streletskaya Sloboda, 20.VI.2009, 32 ex.; Kochkurovo Distr.: Starye Turdaki, 12.VI.2008, 2 ex.

DISTRIBUTION in Mordovia. Temnikov Distr. [Ruchin, Kurmaeva, 2009; Feoktistov, 2011; Orlov et al., 2011; Egorov, Ruchin, 2012, 2013a, 2014; Egorov et al., 2015, 2016, 2017]; Zubova Polyana Distr. [Ruchin, Kurmaeva, 2009]; Torbeevo Distr. [Ruchin, Kurmaeva, 2009]; Bolshie Berezniki Distr. [Timraleev, 1999; Ruchin, 2008a]. Saransk Town [Ruchin, 2008a, 2009]. Tengushevo Distr., Ichalki Distr., Chamzinka Distr., Lyambir Distr., Ruzaevka Distr., Kochkurovo Distr. [all this paper].

BIOLOGY in Mordovia. The main habitats are fringes, glades, felling, roadsides in deciduous and mixed forests. Occurs on inflorescences Anthriscus sylvestris (L.) Hoffm., Aegopodium podagraria L., Leucanthemum vulgare Lam., Pimpinella saxifraga L., Polygonum bistorta L., Anthriscus sylvestris (L.) Hoffm., Filipendula ulmaria (L.) Maxim., Salvia stepposa Schost., Trifolium alpestre L., Rosa sp., Centaurea cyanus L., Plantago lanceolata L. Imago flight lasts from 3rd decade of May to 3rd decade of July.
Cortodera femorata (Fabricius, 1787)

MATERIAL. Temnikov Distr.: MSNR, 18 km NW Temnikov, 3.VI.2017, 1 ex.; MSNR, Pushta, 4.VI.2017, 1 ex., Semishin G.B.

DISTRIBUTION in Mordovia. Temnikov Distr. [Ruchin, 2008b; Ruchin, Kurmaeva, 2009; Egorov et al., 2010; Egorov, Ruchin, 2013a, 2014].

BIOLOGY in Mordovia. Within the Republic of Mordovia it is found only in the reserve and its surroundings. It occurs in ripe pine forests with spruce, birch, aspen and mixed forests. Imago flight lasts from 2 nd decade of May to 3rd decade of June.

\section{Nivellia sanguinosa (Gyllenhal, 1827)}

MATERIAL. Temnikov Distr.: Veselyj, 25.V.2013, 2 ex.; Temnikov Distr.: MSNR, 15 km N Temnikov, 3.VI.2017, 2 ex., Semishin G.B.; Ichalki Distr.: NPS, Lvovskoe forestry, $15 \mathrm{~km}$ NNE Ichalki, 21.V.2008, 2 ex.

DISTRIBUTION in Mordovia. Temnikov Distr. [Egorov, Ruchin, 2012, 2013a; Egorov et al., 2017]. Bolshoe Ignatovo Distr. [Ruchin, 2008a]. Ichalki Distr. [this paper].

BIOLOGY in Mordovia. It was found mainly in alder and mixed forests on the inflorescences of Sorbus aucuparia L., Aegopodium podagraria L., and also on the leaf of Rubus idaeus L. Imago flight lasts from the first decade of May to the 3 rd decade of June.

\section{Alosterna ingrica (Baeckmann, 1902)}

MATERIAL. Temnikov Distr.: MSNR, $15 \mathrm{~km} \mathrm{~N}$ Temnikov, 15-29.VI.2017, 29.VI.2017, 3 ex., Egorov L.V., Semishin G.B.

DISTRIBUTION in Mordovia. Temnikov Distr. [Egorov, Ruchin, 2012; Egorov et al., 2016, 2017]; Ichalki Distr. [Ruchin et al., 2009; Ruchin, Egorov, 2013].

BIOLOGY in Mordovia. It occurs in mixed forests on glades, fringes, cuttings. Noticed on the inflorescences of Anthriscus sylvestris (L.) Hoffm., Aegopodium podagraria L. Imago flight lasts from 1st to 3rd decade of June.

\section{Alosterna tabacicolor (DeGeer, 1775)}

MATERIAL. Temnikov Distr.: MSNR, $15 \mathrm{~km} \mathrm{~N}$ Temnikov, 3.VI.2017, 1 ex., Semishin G.B.; 2.VII.2017, 2 ex.; Temnikov Distr.: Babeevo, 8.VI.2013, 2 ex.; Atyurjevo Distr.: Mordovskaya Kozlovka, 8.VI.2013, 1 ex.; Ichalki Distr.: Khanineevka, 12.VI.2009, 8 ex.; NPS, Barakhmanovskoe forestry, $13 \mathrm{~km} \mathrm{NNE}$ Ichalki, 13.VI.2009, 5 ex.; Kadoshkino Distr.: Latyshovka, 4.VI.2016, 1 ex.; Insar Distr.: Vasina Polyana, 4.VI.2016, 8 ex.; Kochetovka, 4.VI.2016, 3 ex.; Ruzaevka Distr.: Levzhensky, 9.VI.2009, 35 ex.; 3 km N Streletskaya Sloboda, 20.VI.2009, 41 ex.; Palaevka, 26.VI.2016, 1 ex.; Bolshie Berezniki Distr.: Nerlej, 5.VI.2016, 1 ex.

DISTRIBUTION in Mordovia. Temnikov Distr. [Ruchin, 2008b; Ruchin, Kurmaeva, 2009; Egorov, Ruchin, 2012, 2013a, 2014; Egorov et al., 2015, 2016, 2017]; Zubova Polyana Distr. [Ruchin, 2008b; Ruchin, Kurmaeva, 2009]; Atyurjevo Distr. [Ruchin, 2008b; Ruchin, Kurmaeva, 2009]; Ichalki Distr. [Ruchin, 2008b]; Chamzinka Distr. [Ruchin, 2008b]; Kochkurovo Distr. [Ruchin, 2008b]. Saransk Town [Ruchin, 2009]. Kadoshkino Distr., Insar Distr., Ruzaevka Distr., Bolshie Berezniki Distr. [all this paper].

BIOLOGY in Mordovia. It occurs annually in deciduous, mixed forests, pine forests, aspen forests, where it lives on glades, fringes, fellings, clearings, roadsides. It is often noted on inflorescences of Leucanthemum vulgare Lam., Aegopodium podagraria L., Achillea millefolium L., Anthriscus sylvestris (L.) Hoffm., Trifolium alpestre L., Polygonum bistorta L., Pimpinella saxifraga L., Centaurea cyanus L. Imago flight lasts from 3rd decade of May to 3rd decade of June. 
Pseudovadonia livida (Fabricius, 1776)

In Mordovia there is a subspecies $P$. livida bicarinata (Arnold, 1869).

MATERIAL. Krasnoslobodsk Distr.: Krasnoslobodsk, 5.VI.2009, 7 ex.; Staroe Shajgovo Distr.: Nikolskaya Salovka, 8.VII.2017, 4 ex.; Ichalki Distr.: NPS, Lvovskoe forestry, $15 \mathrm{~km}$ NNE Ichalki, 21.VI.2008, 24.VI.2008, 4 ex.; NPS, Barakhmanovskoe forestry, $12 \mathrm{~km}$ NE Ichalki, 21.VII.2009, 1 ex.; Chamzinka Distr. Gorbunovka, 24.VI.2016, 2 ex.; Dubenki Distr.: 8 km SE Engalychevo 1.VII.2009, 20 ex.; Ruzaevka Distr.: Levzhensky, 21.VI.2009, 2 ex

DISTRIBUTION in Mordovia. Temnikov Distr. [Plavilshchikov, 1964; Ruchin, Kurmaeva, 2009; Egorov, Ruchin, 2012, 2013a; Egorov et al., 2015, 2016]; Zubova Polyana Distr. [Ruchin, Kurmaeva, 2009]. Bolshie Berezniki Distr. [Timraleev, 1999]. Krasnoslobodsk Distr., Ichalki Distr., Chamzinka Distr., Dubenki Distr., Ruzaevka Distr. [all this paper].

BIOLOGY in Mordovia. It is common in glades, fringes of mixed and deciduous forests, steppe slopes. Noticed on inflorescences of Achillea millefolium L. s.l., Polygonum bistorta L., Anthriscus sylvestris (L.) Hoffm., Knautia arvensis (L.) Coult. Imago flight lasts from the first decade of June to the 3rd decade of July.

\section{Anoplodera sexguttata (Fabricius, 1775)}

MATERIAL. Temnikov Distr.: MSNR, $15 \mathrm{~km} \mathrm{~N}$ Temnikov, 14.VI.2017, 15.VI.2017, 2 ex.; same label, 29.VI.2017, 1 ex., Semishin G.B.; Ichalki Distr.: Khanineevka, 12.VI.2009, 1 ex.; NPS, Lvovskoe forestry, $15 \mathrm{~km}$ NNE Ichalki, 9.VI.2017, 1 ex., Semishin G.B.; Chamzinka Distr.: Komsomolskij, 22.VI.2008, 1 ex., Ryzhov M.K.; Insar Distr.: Vasina Polyana, 4.VI.2016, 2 ex.; Ruzaevka Distr.: Levzhensky, 9.VI.2009, 3 ex.; 3 km N Streletskaya Sloboda, 20.VI.2009, 3 ex., Saransk, 20.VI.2009, 1 ex

DISTRIBUTION in Mordovia. Temnikov Distr. [Ruchin, 2008b; Ruchin, Kurmaeva, 2009; Orlov et al., 2011; Egorov, Ruchin, 2012; Egorov et al., 2016, 2017]; Zubova Polyana Distr. [Ruchin, 2008b; Ruchin, Kurmaeva, 2009]. Ichalki Distr., Chamzinka Distr., Insar Distr., Ruzaevka Distr., Saransk Town [all this paper].

BIOLOGY in Mordovia. Commonly found in deciduous and mixed forests along the fringes, glades, fellings. Noticed on inflorescences Pimpinella saxifraga L., Polygonum bistorta L., Aegopodium podagraria L., Achillea millefolium L., Anthriscus sylvestris (L.) Hoffm. Imago flight lasts from the 3rd decade of May to the 3rd decade of June.

\section{Stictoleptura maculicornis (DeGeer, 1775)}

MATERIAL. Temnikov Distr.: Tarkhany, 5.VI.2009, 3 ex.; Alkaevo, 30.V.2015, 1 ex.; Veselyj, 6.VI.2015, 1 ex.; MSNR, 15 km N Temnikov, 14.VI.2017, 29.VI.2017, 15-29.VI.2017, 5 ex., Egorov L.V., Semishin G.B.; 2.VII.2017, 2 ex.; 22 km N Temnikov, 4.VII.2017, 1 ex.; $31 \mathrm{~km}$ NE Temnikov, 13.VII.2017, 7 ex.; $18 \mathrm{~km}$ NW Temnikov, 15.VII.2017, 1 ex.; 22 km N Temnikov, 4.VII.2017, 3 ex.; same label, 4.VIII.2017, 1 ex., Semishin G.B.; Atyurjevo Distr.: Russkaya Velyazma, 2.VII.2016, 1 ex.; Strelnikovo, 2.VII.2016, 1 ex.; Elniki Distr.: Novaya Yamskaya Sloboda 22.VII.2017, 1 ex.; Krasnoslobodsk Distr.: Krasnoslobodsk, 5.VI.2009, 7 ex; $2 \mathrm{~km}$ N Selishchi, 5.VI.2009, 4 ex; $5 \mathrm{~km}$ VE Staraya Avgura, 8.VII.2017, 3 ex.; Staroe Shajgovo Distr.: Nikolskaya Salovka, 8.VII.2017, 1 ex.; Ichalki Distr.: Sosnovka, 3.VII.2008, 1 ex.; Khanineevka, 12.VI.2009, 9 ex.; NPS, Barakhmanovskoe forestry, $12 \mathrm{~km}$ NE Ichalki, 14.VI.2009, 25.VII.2017, 3 ex.; NPS, Barakhmanovskoe forestry, $13 \mathrm{~km}$ NNE Ichalki, 13.VI.2009, 1 ex.; NPS, Lvovskoe forestry, $15 \mathrm{~km}$ NNE Ichalki, 17.VI.2008, 18.VII.2008, 3 ex.; NPS, Kemlyanskoe forestry, 7 km N Ichalki, 27.VII.2017, 3 ex.; Chamzinka Distr.: Bolshoe Maresevo, 13.VI.2008, 2 ex.; Ardatov Distr.: Probuzhdenie, 2.VII.2008, 2 ex.; Ruzaevka Distr.: Tatarskaya Pishlya, 30.VI.2008, 1 ex., Kurmaeva
D.K.; 3 km N Streletskaya Sloboda, 20.VI.2009, 3 ex.; Levzhensky, 21.VI.2009, 3 ex.; Palaevka, 26.VI.2016, 3 ex.; Yakovshchina, 26.VI.2016, 1 ex.; Bolshie Berezniki Distr.: 6 km SE Permisi, 12.VI.2015, 2 ex.; Kochkurovo Distr.: Starye Turdaki, 12.VI.2008, $1 \mathrm{ex}$.

DISTRIBUTION in Mordovia. Temnikov Distr. [Ruchin, Kurmaeva, 2009; Feoktistov, 2011; Orlov et al., 2011; Egorov, Ruchin, 2012, 2013a, 2014; Egorov et al., 2015, 2016, 2017]; Zubova Polyana Distr. [Ruchin, Kurmaeva, 2009]; Torbeevo Distr. [Ruchin, Kurmaeva, 2009]. Saransk Town [Ruchin, 2008a, 2009]. Atyurjevo Distr., Elniki Distr., Krasnoslobodsk Distr., Staroe Shajgovo Distr., Ichalki Distr., Chamzinka Distr., Ardatov Distr., Ruzaevka Distr., Bolshie Berezniki Distr., Kochkurovo Distr. [all this paper].

BIOLOGY in Mordovia. Common habitats are glades, clearings, roadsides, fellings in mixed and deciduous forests. It visits inflorescences of Leucanthemum vulgare Lam., Filipendula ulmaria (L.) Maxim., Aegopodium podagraria L., Anthriscus sylvestris (L.) Hoffm., Pimpinella saxifraga L., Geranium pratense L., Polygonum bistorta L., Plantago major L., Achillea millefolium L. s.1., Plantago media L., Fragaria viridis (Duch.) Weston, Fragaria vesca L., Galium aparine L. Imago flight lasts from the 1st decade of June to the 1st decade of August.

\section{Stictoleptura rubra (Linnaeus, 1758)}

MATERIAL. Tengushevo Distr.: Khlebino, 2.VIII.2014, 1 ex.; Temnikov Distr.: Temnikov, 25.VII.2009, 1 ex.; MSNR, 15 km N Temnikov, 5.VIII.2017, 1 ex.; MSNR, 22 km N Temnikov, 4.VIII.2017, 6.VIII.2017, 7 ex., Semishin G.B.; MSNR, 25 km E Temnikov, 7.VIII.2017, 2 ex., Bolshakov L.V.; same label, 6.VIII.2017, 5 ex.; MSNR, 43 km NE Temnikov, 1.VIII.2017, 1 ex.; MSNR, 18 km NNE Temnikov, 4.VIII.2017, 7.VIII.2017, 2 ex., Semishin G.B.; MSNR, 15 km N Temnikov, 6-24.VIII.2017, 1 ex., Egorov L.V., Semishin G.B.; MSNR, Pushta, 9.IX.2017, 1 ex., Egorov L.V.; Zubova Polyana Distr.: Kiselevka, 30.VII.2009, 5+, $10^{7}$; Lesnoj, 2.VIII.2015, 1 ex.; Atyurjevo Distr.: Strelnikovo, 2.VII.2016, 1 ex.; Elniki Distr.: Novye Shaly, 19.VII.2015, 1 ex.; Kovylkino Distr: Andreevka, 30.VII.2008, $20^{7}+2$ ㅇ. Krasnoslobodsk Distr.: Sivin, 20.VII.2017, 1 ex.; Staroe Shajgovo Distr.: Lesnichestvo, 17.VII.2009, 2 ex.; Ichalki Distr.: NPS, Lvovskoe forestry, $15 \mathrm{~km}$ NNE Ichalki, 17.VI.2008, 1 ex.; NPS, Barakhmanovskoe forestry, $12 \mathrm{~km}$ NE Ichalki, 21.VII.2009, 25.VII.2017, 3 ex.; NPS, Barakhmanovskoe forestry, $13 \mathrm{~km}$ NNE Ichalki, 1430.VIII.2017, 3 ex., Semishin G.B.; Bolshie Berezniki Distr.: Simkinskoe lesnichestvo, 12.VIII.2017, 4 ex.; 9 km S Simkino, 2.VII.2009, 2 ex., Kurmaeva D.K.; 5 km SE Permisi, 24.VII.2008, 1 ex., Kurmaeva D.K.; Kochkurovo Distr.: Mordovskoe Davydovo, 23.VII.2008, 107, 19, Kurmaeva D.K.; Muran, 29.VII.2017, 1 ex.

DISTRIBUTION in Mordovia. Temnikov Distr. [Plavilshchikov, 1964; Ruchin, Kurmaeva, 2009; Orlov et al., 2011; Egorov, Ruchin, 2012, 2013a; Egorov et al., 2015, 2016, 2017]; Zubova Polyana Distr. [Ruchin, Kurmaeva, 2009]; Krasnoslobodsk Distr. [Ruchin, Kurmaeva, 2009]; Ichalki Distr. [Ruchin, 2008a; Ruchin, Grishutkin, 2008]; Bolshoe Ignatovo Distr. [Ruchin, Grishutkin, 2008]; Bolshie Berezniki Distr. [Timraleev, 1999]. Saransk Town [Ruchin, 2008a, 2009]. Tengushevo Distr., Atyurjevo Distr., Elniki Distr., Kovylkino Distr., Staroe Shajgovo Distr., Kochkurovo Distr. [all this paper].

BIOLOGY in Mordovia. It is often observed in mixed forests, pine forests, roadside wood lines with an admixture of pine. Often found on young shoots of pines, fallen pine trees. Imago occur on inflorescences of Chamaenerion angustifolium (L.) Scop., Filipendula ulmaria (L.) Maxim., Pimpinella saxifraga L., Angelica sylvestris L., Centaurea cyanus L., Cichorium intybus L. Imago flight lasts from 1st decade of June to 1 st decade of September. 
Stictoleptura variicornis (Dalman, 1817)

MATERIAL. Temnikov Distr.: MSNR, 18 km NNE Temnikov, 7.VIII.2017, 1 ex., Semishin G.B.

DISTRIBUTION in Mordovia. Temnikov Distr. [Plavilshchikov, 1964; Egorov, Ruchin, 2012, 2013a; Egorov et al., 2017].

BIOLOGY in Mordovia. Rare species. Within the republic it was discovered only in Mordovia reserve, where it was found in mixed forests, pine forests on umbelliferae plants. Imago flight lasts from 1st decade of July to 1st decade of August.

\section{Anastrangalia reyi (Heyden, 1889)}

MATERIAL. Temnikov Distr.: Veselyj, 6.VI.2015, 1 ex.; MSNR, 18 km N Temnikov, 20.V.2017, 1 ex.; 18 km NNE Temnikov, 18.VI.2017, 1 ex.; 31 km NE Temnikov, 13.VII.2017, 2 ex.; 21 km NW Temnikov, 15.VII.2017, 2 ex.; 25 km E Temnikov, 6.VIII.2017, 1 ex.; Atyurjevo Distr.: Kamenka, 21.VI.2016, 1 ex.; Ichalki Distr. NPS, Barakhmanovskoe forestry, $12 \mathrm{~km}$ NE Ichalki, 14.VI.2009, 25.VII.2017, 3 ex.; NPS, Barakhmanovskoe forestry, $13 \mathrm{~km} \mathrm{NNE}$ Ichalki, 13.VI.2009, 1 ex.; Kochkurovo Distr.: Starye Turdaki, 12.VI.2008, 2 ex.

DISTRIBUTION in Mordovia. Temnikov Distr. [Orlov et al., 2011; Egorov, Ruchin, 2012, 2013a, 2014; Egorov et al., 2015, 2016, 2017]; Zubova Polyana Distr. [Ruchin, Kurmaeva, 2009]; Torbeevo Distr. [Ruchin, Kurmaeva, 2009]; Ichalki Distr. [Ruchin, 2008b]. Saransk Town [Ruchin, 2008b, 2009; Semishin, 2009]. Atyurjevo Distr., Kochkurovo Distr. [all this paper].

BIOLOGY in Mordovia. It inhabits deciduous and mixed forests. Imago visit inflorescences of Angelica sylvestris L., Aegopodium podagraria L., Leucanthemum vulgare Lam., Cichorium intybus L. Imago flight lasts from 2nd decade of May to 1st decade of August.

\section{Anastrangalia sanguinolenta (Linnaeus, 1760)}

MATERIAL. Temnikov Distr.: Bochino, VII.2008, 2 ex., Susarev S.V.; Veselyj, 6.VI.2015, 1 ex.; MSNR, 18 km N Temnikov, 4.VII.2017, 1 ex.; $31 \mathrm{~km}$ NE Temnikov, 13.VII.2017, 6 ex.; $22 \mathrm{~km}$ N Temnikov, 4.VIII.2017, 3 ex., Semishin G.B.; Ichalki Distr.: NPS, Kemlyanskoe forestry, 4 km NNE Ichalki, 21.VII.2009, 1 ex.; NPS, Barakhmanovskoe forestry, $12 \mathrm{~km}$ NE Ichalki, 25.VII.2017, 5 ex.

DISTRIBUTION in Mordovia. Temnikov Distr. [Mozolevskaya et al., 1971; Ruchin, Kurmaeva, 2009; Egorov, Ruchin, 2012, 2014; Egorov et al., 2015, 2016, 2017]; Zubova Polyana Distr. [Ruchin, Kurmaeva, 2009]; Torbeevo Distr. [Ruchin, Kurmaeva, 2009]; Ichalki Distr. [Ruchin, 2008b]. Ichalki Distr. [this paper].

BIOLOGY in Mordovia. Found in mixed forests on inflorescences of Achillea millefolium L. s.l., Aegopodium podagraria L., Leucanthemum vulgare Lam. Imago flight lasts from the 3rd decade of May to the 1st decade of August.

\section{Lepturobosca virens (Linnaeus, 1758)}

MATERIAL. Temnikov Distr.: 5 km NE Purdoshki, VI.2008, 3 ex., Susarev S.V.; MSNR, 18 km NNE Temnikov, 18.VI.2017, 1 ex.; same label, VII.2008, 1 ex., Susarev S.V.; same label, 4.VII.2017, 2 ex.; MSNR, 18 km N Temnikov, 11.VII.2017, 1 ex.; MSNR, 31 km NE Temnikov, 13.VII.2017, 3 ex.; MSNR, 21 km NW Temnikov, 15.VII.2017, 1 ex.; MSNR, $22 \mathrm{~km} \mathrm{~N}$ Temnikov, 4.VII.2017, 16.VII 2017, 1 ex.; same label, 4.VIII.2017, 1 ex., Semishin G.B.; MSNR, 43 km NE Temnikov, 1.VIII.2017, 2 ex.; MSNR, 25 km E Temnikov, 6.VIII.2017, 3 ex.; Elniki Distr.: Novye Shaly, 19.VII.2015, 1 ex.; Malye Mordovskie Poshaty, 22.VII.2017, 1 ex.; Kovylkino Distr.: Andreevka, 30.VII.2008, 1 ex.; Staroe Shajgovo Distr.: Lesnichestvo, 17.VII.2009, 1 ex.; Ichalki Distr.: NPS, Barakhmanovskoe forestry, 12 km NE Ichalki, 14.VI.2009, 1 ex.; 21.VII.2009, 25.VII.2017, 4 ex.; NPS, Kemlyanskoe forestry, $7 \mathrm{~km} \mathrm{~N}$ Ichalki, 27.VII.2017, 2 ex.; Kochkurovo Distr.: Muran, 29.VII.2017, 1 ex.
DISTRIBUTION in Mordovia. Temnikov Distr. [Plavilshchikov, 1964; Ruchin, Kurmaeva, 2009; Orlov et al., 2011; Egorov, Ruchin, 2012, 2013a, 2014; Egorov et al., 2015, 2016, 2017]; Krasnoslobodsk Distr. [Ruchin, Kurmaeva, 2009]; Ichalki Distr. [Ruchin, 2008a; Ruchin, Grishutkin, 2008]. Elniki Distr., Kovylkino Distr., Staroe Shajgovo Distr., Kochkurovo Distr. [all this paper].

BIOLOGY in Mordovia. It occurs in mixed forests, where it prefers fringes, glades, roadsides. Common in the flooded meadows. It visits inflorescences of Angelica sylvestris L., Filipendula ulmaria (L.) Maxim., Leucanthemum vulgare Lam., Aegopodium podagraria L., Conyza canadensis (L.) Cronq., Heracleum sosnowskyi Manden, Pimpinella saxifraga L., Conium maculatum L. Imago flight lasts from 3rd decade of May to 3rd decade of August.

\section{Etorofus pubescens (Fabricius, 1787)}

DISTRIBUTION in Mordovia. Tengushevo Distr. [Ruchin et al., 2014, 2015, 2016]. Temnikov Distr. [Plavilshchikov, 1964; Orlov et al., 2011; Egorov, Ruchin, 2012, 2013a, 2014; Ruchin et al., 2010, 2014, 2015, 2016, 2017]; Zubova Polyana Distr. [Ruchin, Kurmaeva, 2009; Ruchin et al., 2010].

BIOLOGY in Mordovia. It occurs in mixed forests on inflorescences of Anthriscus sylvestris (L.) Hoffm., Aegopodium podagraria $\mathrm{L}$. Imago flight lasts from the 1st decade of June to the 2 nd decade of July.

\section{Judolia sexmaculata (Linnaeus, 1758)}

MATERIAL. Temnikov Distr.: 3 km NW Purdoshki, VII.2009, 1 ex., Susarev S.V.; MSNR, 15 km N Temnikov, 1-15.VI.2017, 2 ex., Egorov L.V., Semishin G.B.; Ichalki Distr.: NPS, Lvovskoe forestry, $15 \mathrm{~km}$ NNE Ichalki, 9.VI.2017, 1 ex., Semishin G.B.

DISTRIBUTION in Mordovia. Temnikov Distr. [Feoktistov, 2011; Orlov et al., 2011; Ruchin, Kurmaeva, 2009; Egorov, Ruchin, 2012, 2013a; Egorov et al., 2016, 2017]. Ichalki Distr. [this paper].

BIOLOGY in Mordovia. Within the republic it was found only in Mordovia Reserve and NP Smolny and their surroundings. Noticed in mixed forests on inflorescences of Rubus idaeus L., Polygonum bistorta L. Imago flight lasts from the 3rd decade of May to the 1st decade of July.

\section{Pachytodes erraticus (Dalman, 1817)}

MATERIAL. Chamzinka Distr.: Komsomolskij, 21.VIII.2009, 2 ex., Ryzhov M.K.; Kochkurovo Distr.: Mordovskoe Davydovo, 24.VI.2016, 1 ex.; Krasnaja Zorka, 29.VII.2017, 1 ex.

DISTRIBUTION in Mordovia. Ruzaevka Distr., Bolshie Berezniki Distr. [Ruchin, 2008b]. Chamzinka Distr., Kochkurovo Distr. [all this paper].

BIOLOGY in Mordovia. It occurs in deciduous and mixed forests of the eastern part of the republic. Imago found on the inflorescences of Pimpinella saxifraga L., Achillea millefolium L. s.l. Imago flight lasts from the $3 \mathrm{rd}$ decade of June to the 3rd decade of August.

\section{Oedecnema gebleri Ganglbauer, 1889}

MATERIAL. Temnikov Distr.: MSNR, $22 \mathrm{~km} \mathrm{~N}$ Temnikov, 5.V.2016, 1 ex.; MSNR, $32 \mathrm{~km} \mathrm{~N}$ Temnikov, 29.V.2016, 1 ex.; MSNR, 18 km NNE Temnikov, 3.VI.2016, 1 ex.; 18 km NNE Temnikov, 18.VI.2017, 1 ex.; Zubova Polyana Distr.: Lesnoj, 18.VI. 2011, 1 ex., Artaev O.N.

DISTRIBUTION in Mordovia. Lyambir Distr. [Ruchin et al., 2009]. Temnikov Distr., Zubova Polyana Distr. [all this paper].

BIOLOGY in Mordovia. Single specimens were found in mixed, deciduous and pine forests. In 2016, it was discovered 
immediately in three localities on the territory of Mordovia reserve. It is possible that the number of this species within the reserve has risen at that moment. Imago was once recorded on Taraxacum officinale Wigg.

\section{Leptura aethiops Poda von Neuhaus, 1761}

MATERIAL. Temnikov Distr.: Tarkhany, 5.VI.2009, 8 ex.; Ichalki Distr.: Khanineevka, 12.VI.2009, 1 ex.; NPS, Barakhmanovskoe forestry, $12 \mathrm{~km}$ NE Ichalki, 14.VI.2009, 1 ex.; Ardatov Distr.: Oktyabrsky, 19.V.2008, 1 ex.; Kochkurovo Distr.: Starye Turdaki, 12.VI.2008, 1 ex.; Saransk, 20.VI.2009, 1 ex.

DISTRIBUTION in Mordovia. Temnikov Distr. [Orlov et al., 2011]; Ichalki Distr. [Ruchin, 2008a; Ruchin, Grishutkin, 2008]. Ardatov Distr., Kochkurovo Distr. [all this paper].

BIOLOGY in Mordovia. It occurs in mixed and deciduous forests along the fringes and glades. The imago was recorded on Plantago media L. Imago flight lasts from the 2nd decade of May to the 3rd decade of June.

\section{Leptura annularis Fabricius, 1801}

MATERIAL. Temnikov Distr.: MSNR, $15 \mathrm{~km} \mathrm{~N}$ Temnikov, 15-29.VI.2017, 3 ex., Egorov L.V., Semishin G.B.; 31 km NE Temnikov, 13.VII.2017, 1 ex.

DISTRIBUTION in Mordovia. Temnikov Distr. [Plavilshchikov, 1964; Ruchin, Kurmaeva, 2009; Orlov et al., 2011; Egorov, Ruchin, 2012, 2013a, 2014; Egorov et al., 2016, 2017]. Ichalki Distr. [Ruchin, 2008a; Ruchin, Grishutkin, 2008]. Bolshie Berezniki Distr. [Timraleev, 1999].

BIOLOGY in Mordovia. Commonly found on the territory of Mordovia reserve. Habitats are mixed and deciduous forests. Imago visit inflorescences of Dactylorhiza fuchsii (Druce.) Soo, Anthriscus sylvestris (L.) Hoffm., Aegopodium podagraria L., Cichorium intybus L., Carum carvi L., Polygonum bistorta L. Imago flight lasts from the 1st decade of June to the 3rd decade of July.

\section{Leptura aurulenta Fabricius, 1793}

DISTRIBUTION in Mordovia. Temnikov Distr. [Ruchin, Egorov, 2018].

BIOLOGY in Mordovia. The only finding in the republic. Female of $L$. aurulenta was caught in the quarter 324 of Mordovia Reserve on the western clearing. The beetle was found on the inflorescence of a plant from the Umbelliferae family. It is the third confirmed registration of a very rare species of Russian fauna, and this is the most eastern finding of a taxon within the range.

\section{Leptura quadrifasciata Linnaeus, 1758}

MATERIAL. Temnikov Distr.: Bochino, VII.2008, 1 ex., Susarev S.V.; MSNR, Mikhajlovka, VI-VII.2012, 1 ex.; Temnikov Distr.: MSNR, 16 km N Temnikov, VI-VII.2012, 1 ex.; MSNR, 18 km NNE Temnikov, 18.VI.2017, 1 ex.; MSNR, 15 km N Temnikov, 2.VII.2017, 27.VII.-6.VIII.2017, 15 ex.; 13.VII.2017, 1 ex., Semishin G.B.; MSNR, 31 km NE Temnikov, 13.VII.2017, 2 ex.; MSNR, 21 km NW Temnikov, 13.VII.2017, 1 ex; MSNR, 22 km N Temnikov, 16.VII.2017, 5.VIII.2017, 6.VIII.2017, 6 ex.; MSNR, 43 km NE Temnikov, 1.VIII.2017, 2 ex.; MSNR, 25 km E Temnikov, 6.VIII.2017, 1 ex.; same label, 7.VIII.2017, 2 ex., Bolshakov L.V.; Zubova Polyana Distr.: Kargashino, 29.VII.2009, 1 ex.; Atyurjevo Distr.: Dmitriev Usad, VI-VII.2012, 2 ex.; Elniki Distr.: Malye Mordovskie Poshaty, 22.VII.2017, 1 ex.; Staroe Shajgovo Distr.: Lesnichestvo, 17.VII.2009, 8 ex.; Ichalki Distr.: NPS, Kemlyanskoe forestry, $7 \mathrm{~km}$ N Ichalki, 27.VII.2017, 3 ex.; Ruzaevka Distr.: Levzhensky, 22.VII.2008, 1 ex.; Krasnoslobodsk Distr.: Staroe Sindrovo, 1.VIII.2008, 1 ex.; Kochkurovo Distr.: Muran, 29.VII.2017, 1 ex.

DISTRIBUTION in Mordovia. Temnikov Distr. [Plavilshchikov, 1964; Ruchin, Kurmaeva, 2009; Orlov et al., 2011;
Egorov, Ruchin, 2012, 2013a, 2014; Egorov et al., 2015, 2016, 2017]; Zubova Polyana Distr., Torbeevo Distr., Krasnoslobodsk Distr. [Ruchin, Kurmaeva, 2009]; Ichalki Distr. [Ruchin, 2008a; Ruchin, Grishutkin, 2008]; Bolshie Berezniki Distr. [Timraleev, 1999]. Atyurjevo Distr., Elniki Distr., Staroe Shajgovo Distr., Ruzaevka Distr., Kochkurovo Distr. [all this paper].

BIOLOGY in Mordovia. It is commonly found in open biotopes of mixed, broad-leaved and small-leaved forests, sometimes on floodplain meadows in birch-aspen groves, aspen or birch plantations. Imago often occur on the inflorescences of Achillea millefolium L. s.1., Aegopodium podagraria, Heracleum sosnowskyi Manden, Leucanthemum vulgare Lam., Cirsium arvense (L.) Scop., Filipendula ulmaria (L.) Maxim., Angelica sylvestris L., Tanacetum vulgare L. Imago flight lasts from the first decade of June to the 2nd decade of August.

\section{Leptura thoracica Creutzer, 1799}

MATERIAL. Temnikov Distr.: Bochino, VII.2008, 1 ex., Susarev S.V.; MSNR, 25 km E Temnikov, 6.VIII.2017, 1 ex.

DISTRIBUTION in Mordovia. Temnikov Distr. [Mozolevskaya et al., 1971; Egorov, Ruchin, 2012, 2014; Egorov et al., 2015, 2016].

BIOLOGY in Mordovia. Within the Republic of Mordovia it is found only in the reserve and its surroundings. Occurs in ripe pine forests with spruce, birch, aspen. Imago flight lasts from the 1st decade of May to the 1st decade of August.

\section{Lepturalia nigripes (DeGeer, 1775)}

MATERIAL. Temnikov Distr.: MSNR, 18 km NNE Temnikov, 18.VI.2017, 1 ex.; MSNR, 31 km NE Temnikov, 13.VII.2017, 2 ex.; MSNR, 15 km N Temnikov, 13.VII.2017, 1 ex., Semishin G.B.; Kochkurovo Distr.: Mordovskoe Davydovo, 25.VI.2016, 1 ex.; Ichalki Distr.: NPS, Kemlyanskoe forestry, $7 \mathrm{~km} \mathrm{~N}$ Ichalki, 27.VII.2017, 1 ex.; NPS, Lvovskoe forestry, $15 \mathrm{~km}$ NNE Ichalki, 24.VII.2008, 1 ex.

DISTRIBUTION in Mordovia. Temnikov Distr. [Plavilshchikov, 1964; Orlov et al., 2011; Egorov, Ruchin, 2013a, 2014; Egorov et al., 2015, 2016, 2017]; Bolshoe Ignatovo Distr. [Ruchin, 2008a]. Kochkurovo Distr., Ichalki Distr. [all this paper].

BIOLOGY in Mordovia. Occurs sporadically in mixed and deciduous forests. Imago found on the inflorescences of Aegopodium podagraria L., Leucanthemum vulgare Lam., Anthriscus sylvestris (L.) Hoffm. Imago flight lasts from the 3rd decade of May to the 3rd decade of July.

\section{Strangalia attenuata (Linnaeus, 1758)}

MATERIAL. Tengushevo Distr.: Barashevo, 18.VII.2015, 1 ex.; Klemeshchej, 18.VII.2015, 1 ex.; Temnikov Distr.: Temnikov, 25.VII.2009, 1 ex.; MSNR, $31 \mathrm{~km}$ NE Temnikov, 13.VII.2017, 1 ex.; $21 \mathrm{~km}$ NW Temnikov, 13.VII.2017, 2 ex.; 18 km NW Temnikov, 5.VIII.2017, 2 ex.; 22 km N Temnikov, 5.VIII.2017, 6.VIII.2017, 3 ex.; 25 km E Temnikov, 7.VIII.2017, 2 ex.; 18 km NNE Temnikov, 7.VIII.2017, 1 ex., Bolshakov L.V.; $15 \mathrm{~km} \mathrm{~N}$ Temnikov, 5. VIII.2017, 27.VII.-6.VIII.2017, 2 ex., Egorov L.V., Semishin G.B.; Atyurjevo Distr.: Strelnikovo, 2.VII.2016, 1 ex.; Elniki Distr.: Novye Shaly, 19.VII.2015, 1 ex.; Malye Mordovskie Poshaty, 19.VII.2015, 22.VII.2017, 2 ex.; Novaya Yamskaya Sloboda, 22.VII.2017, 1 ex.; Kovylkino Distr.: Andreevka, 30.VII.2008, 1 ex.; Staroe Shajgovo Distr.: Lesnichestvo, 17.VII.2009, 8 ex.; Insar Distr.: Novlej, 19.VIII. 2017, 1 ex.; Ichalki Distr.: Khanineevka, 23.VII.2008, 1 ex.; NPS, Barakhmanovskoe forestry, $12 \mathrm{~km}$ NE Ichalki, 21.VII.2009, 25.VII. 2017, 10 ex.; NPS, Kemlyanskoe forestry, 7 km N Ichalki, 27.VII. 2017, 3 ex.; Ardatov Distr.: Probuzhdenie, 2.VII.2008, 2 ex.; 3 km NW Sosnovoe, 18.VIII.2008, 1 ex.; Dubenki Distr.: 8 km SE Engalychevo 1.VII.2009, 4 ex.; Lyambir Distr.: Belogorskoe, 29.VII.2016, 1 ex.; Ruzaevka Distr.: Palaevka, 26.VI.2016, 2 ex.; 3 km N Stre- 
letskaya Sloboda, 20.VI.2009, 1 ex.; Levzhensky, 21.VI.2009, 1 ex.; Tatarskaya Pishlya, 30.VI.2008, 1 ex., D.K. Kurmaeva; Levzhensky, 22.VII.2008, 1 ex.; Bolshie Berezniki Distr.: Gart, 19.VII.2008, 1 ex.; Kochkurovo Distr.: Mordovskoe Davydovo, 23.VII.2008, 1 ex., Kurmaeva D.K.; Starye Turdaki, 8.VII.2008, 1 ex.; 5 km E Sabaevo, 25.VI.2016, 1 ex.; Novaya Pyrma, 11.VIII.2017, 1 ex.; Krasnaja Zorka, 29.VII.2017, 1 ex.; Muran, 29.VII.2017, 3 ex.

DISTRIBUTION in Mordovia. Temnikov Distr. [Mozolevskaya et al., 1971; Ruchin, Kurmaeva, 2009; Orlov et al., 2011; Egorov, Ruchin, 2012, 2013a, 2014; Egorov et al., 2015, 2016, 2017]; Zubova Polyana Distr., Krasnoslobodsk Distr. [Ruchin, Kurmaeva, 2009]; Ichalki Distr. [Ruchin, 2008a; Ruchin, Grishutkin, 2008]; Bolshie Berezniki Distr. [Ruchin, 2008a]. Saransk Town [Ruchin, 2009]. Tengushevo Distr., Atyurjevo Distr., Elniki Distr., Kovylkino Distr., Staroe Shajgovo Distr., Insar Distr., Ardatov Distr., Dubenki Distr., Lyambir Distr., Ruzaevka Distr. [all this paper].

BIOLOGY in Mordovia. It is commonly found in mixed, broad-leaved, small-leaved forests, pine forests, forest plantations. The biotopes are small meadows near the forest tracts, glades, fringes. Imago occur on inflorescences of Aegopodium podagraria L., Filipendula ulmaria (L.) Maxim., Angelica sylvestris L., Pimpinella saxifraga L., Anthriscus sylvestris (L.) Hoffm., Selinum carvifolia (L.) L., Viburnum opulus L., Phleum pratense L., Solidago canadensis L., Achillea millefolium L. s.l., Arctium lappa L., Tanacetum vulgare L., Centaurea jacea L., Cichorium intybus L. Imago flight lasts from the 1 st decade of June to the 2 nd decade of August.

Rutpela maculata (Poda von Neuhaus, 1761)

MATERIAL. Tengushevo Distr.: $6 \mathrm{~km}$ W Barashevo, 18.VII.2015, 1 ex.; Barashevo, 18.VII.2015, 1 ex.; Klemeshchej, 18.VII.2015, 1 ex.; Temnikov Distr.: MSNR, 25 km E Temnikov, 6.VIII.2017, 3 ex.; Atyurjevo Distr.: Russkaya Velyazma, 2.VII.2016, 1 ex.; Elniki Distr.: Malye Mordovskie Poshaty, 22.VII.2017, 2 ex.; Staroe Shajgovo Distr.: Lesnichestvo, 17.VII.2009, 28 ex.; Ichalki Distr.: Khanineevka, 12.VI.2009, 23.VII.2008, 2 ex.; NPS, Lvovskoe forestry, $15 \mathrm{~km}$ NNE Ichalki, 14.VII.2006, 23.VI.2008, 25.VI.2008, 27.VI.2008, 30.VI.2008, 22.VII.2017, 8 ex., Semishin G.B.; NPS, Barakhmanovskoe forestry, $12 \mathrm{~km}$ NE Ichalki, 20.VI.2006, 14.VII.2007, 27.VII.2017, 25.VII.2017, 8 ex.; NPS, Kemlyanskoe forestry, 7 km N Ichalki, 27.VII.2017, 5 ex.; Ardatov Distr.: Kozlovka, 2007, 2 ex.; Chamzinka Distr.: Komsomolskij, 22.VI.2008, 1 ex., Ryzhov M.K.; Ruzaevka Distr.: 3 km N Streletskaya Sloboda, 20.VI.2009, 3 ex.; Bolshie Berezniki Distr.: Gart, 19.VII.2008, 1 ex.; Simkinskoe lesnichestvo, 12.VIII.2017, 1 ex.; Kochkurovo Distr.: Starye Turdaki, 8.VII.2008, 1 ex.; Mordovskoe Davydovo, 25.VI.2016, 1 ex.; Muran, 29.VII.2017, 3 ex.; Krasnaja Zorka, 29.VII.2017, 1 ex.; Novaya Pyrma, 11.VIII.2017, 2 ex.

DISTRIBUTION in Mordovia. Temnikov Distr. [Feoktistov, 2011; Orlov et al., 2011; Egorov et al., 2010, 2016; Egorov, Ruchin, 2013a, 2014]; Krasnoslobodsk Distr. [Ruchin, Kurmaeva, 2009]; Ichalki Distr. [Ruchin, 2008a]; Lyambir Distr. [Semishin, 2009]. Saransk Town [Ruchin, 2008a, 2009; Semishin, 2009]. Tengushevo Distr., Atyurjevo Distr., Elniki Distr., Staroe Shajgovo Distr., Ardatov Distr., Chamzinka Distr., Ruzaevka Distr., Bolshie Berezniki Distr., Kochkurovo Distr. [all this paper].

BIOLOGY in Mordovia. It occurs in the same habitats as the previous species. Adult beetles visit inflorescences of Selinum carvifolia (L.) L., Filipendula ulmaria (L.) Maxim., Polygonum bistorta L., Leucanthemum vulgare Lam., Rosa sp., Knautia arvensis (L.) Coult., Aegopodium podagraria L., Angelica sylvestris L., Arctium sp., Pimpinella saxifraga L., Inula britannica L., Tanacetum vulgare L., Carduus sp., Cirsium arvense (L.) Scop., Viburnum opulus L., Chamaenerion angustifolium (L.) Scop., Taraxacum sp. Imago flight lasts from the first decade of June to the 2nd decade of August.

Stenurella bifasciata (O.F. Müller, 1776)

MATERIAL. Tengushevo Distr.: Barashevo, 18.VII.2015, 1 ex.; Temnikov Distr.: Kushki, 2.VII.2016, 1 ex.; Temnikov Distr.: MSNR, $31 \mathrm{~km}$ NE Temnikov, 13.VII.2017, 2 ex.; $21 \mathrm{~km} \mathrm{NW}$ Temnikov, 15.VII.2017, 1 ex.; MSNR, 25 km E Temnikov, 6.VIII. 2017, 1 ex.; MSNR, 18 km NNE Temnikov, 4.VIII.2017, 7.VIII.2017, 2 ex., Semishin G.B.; Atyurjevo Distr.: Mordovskaya Kozlovka, VI-VII.2012, 1 ex.; Pichepolonga, 2.VII.2016, 1 ex.; Kovylkino Distr.: Andreevka, 30.VII.2008, $10^{7}+1$. . Staroe Shajgovo Distr.: Lesnichestvo, 17.VII.2009, 1 ex.; Govorovo, 10.VII.2016, 1 ex.; Ichalki Distr.: Sosnovka, 3.VII.2008, 1 ex.; Khanineevka, 23.VII. 2008, 1 ex.; NPS, Barakhmanovskoe forestry, $12 \mathrm{~km}$ NE Ichalki, 21.VII.2009, 25.VII.2017, 27.VII.2017, 6 ex; NPS, Kemlyanskoe forestry, 4 km NNE Ichalki, 25.VII.2017, 2 ex.; NPS, Kemlyanskoe forestry, $7 \mathrm{~km}$ N Ichalki, 27.VII.2017, 6 ex.; NPS, Barakhmanovskoe forestry, $13 \mathrm{~km}$ NNE Ichalki, 20.VII.-1.VIII.2017, 1 ex., Semishin G.B.; NPS, Lvovskoe forestry, $15 \mathrm{~km}$ NNE Ichalki, 7.VII.2017, 1 ex., Semishin G.B.; Dubenki Distr.: 8 km SE Engalychevo, 1.VII.2009, 14 ex.; Atyashevo Distr.: Atyashevo, 24.VI.2016, 1 ex.; Bolshie Berezniki Distr.: Simkinskoe lesnichestvo, 12.VIII.2017, 1 ex.; Ruzaevka Distr.: Levzhensky, 22.VII.2008, 13.VI.2015, 2 ex.; Kochkurovo Distr.: Novaya Pyrma, 11.VIII.2017, 5 ex.; 5 km E Sabaevo, 25.VI.2016, 1 ex.; Kachelay, 22.VII.2008, 1 ex., Kurmaeva D.K.

DISTRIBUTION in Mordovia. Temnikov Distr. [Plavilshchikov, 1964; Orlov et al., 2011; Ruchin, Kurmaeva, 2009; Egorov, Ruchin, 2012, 2013a, 2014; Egorov et al., 2015, 2016, 2017]; Bolshie Berezniki Distr. [Timraleev, 1999; Ruchin, 2008a]; Ichalki Distr. [Ruchin, 2008a; Ruchin, Grishutkin, 2008]. Saransk Town [Ruchin, 2009]. Tengushevo Distr., Atyurjevo Distr., Kovylkino Distr., Staroe Shajgovo Distr., Dubenki Distr., Atyashevo Distr., Ruzaevka Distr., Kochkurovo Distr. [all this paper].

BIOLOGY in Mordovia. When abundant commonly occurs in mixed, broad-leaved, small-leaved, spruce, pine forests, forest plantations. The biotopes are meadows near woodlands, glades, fringes. Imago occur on inflorescences of Leucanthemum vulgare Lam., Filipendula ulmaria (L.) Maxim., Knautia arvensis (L.) Coult., Cirsium arvense (L.) Scop., Achillea millefolium L. s.l., Conium maculatum L., Inula britannica L., Aegopodium podagraria L., Fragaria vesca L., Angelica sylvestris L., Erigeron canadensis L., Antennaria dioica (L.) Gaertn., Galium aparine L., Alopecurus pratensis L., Calamagrostis arundinacea (L.) Roth. Imago flight lasts from the first decade of June to the 2nd decade of August.

\section{- Stenurella jaegeri (Hummel, 1825)}

REMARK. It is indicated for Mordovia reserve [Mozolevskaya et al., 1971]. Occurs in the Caucasus, including Transcaucasia (Georgia, Armenia, Azerbaijan) [Danilevsky, 2014]. Finding in this region is questionable.

\section{Stenurella melanura (Linnaeus, 1758)}

MATERIAL. Tengushevo Distr.: $6 \mathrm{~km}$ W Barashevo, 18.VII. 2015, 1 ex.; Barashevo, 18.VII.2015, 1 ex.; Temnikov Distr.: 5 km NE Purdoshki, VI.2008, 2 ex., Susarev S.V.; Mikhajlovka, VI-VII. 2012, 1 ex.; Kushki, 2.VII.2016, 1 ex.; Temnikov Distr.: MSNR, 18 km N Temnikov, 4.VII.2017, 3 ex.; $31 \mathrm{~km}$ NE Temnikov, 13.VII. 2017, 6.VIII.2017, 2 ex.; 21 km NW Temnikov, 15.VII.2017, 4 ex.; $25 \mathrm{~km}$ E Temnikov, 6.VIII.2017, 1 ex.; $22 \mathrm{~km} \mathrm{~N}$ Temnikov, 4.VII. 2017, 16.VII.2017, 3 ex.; same label, 4.VIII.2017, 2 ex., Semishin G.B.; Zubova Polyana Distr.: Udevo, 8.VI.2008, 1 ex.; Vysha, 8.VI.2008, 1 ex.; Krutets, 17.VII.2008, 2 ex.; Atyurjevo Distr.: Mordovskaya Kozlovka, VI-VII.2012, 2 ex.; Pichepolonga, 2.VII. 2016, 2 ex.; Elniki Distr.: Novye Shaly, 19.VII.2015, 1 ex.; Novaya 
Yamskaya Sloboda, 22.VII.2017, 2 ex.; Krasnoslobodsk Distr.: 5 km VE Staraya Avgura, 8.VII.2017, 2 ex.; Staroe Shajgovo Distr. Lesnichestvo, 17.VII.2009, 7 ex.; Nikolskaya Salovka, 8.VII.2017, 2 ex.; Ichalki Distr.: Khanineevka, 23.VII.2008, 1 ex.; Sosnovka, 3.VII.2008, 2 ex.; NPS, Barakhmanovskoe forestry, $12 \mathrm{~km} \mathrm{NE}$ Ichalki, 21.VII.2009, 25.VII.2017, 27.VII.2017, 11 ex.; NPS, Kemlyanskoe forestry, $7 \mathrm{~km}$ N Ichalki, 27.VII.2017, 14 ex.; NPS, Barakhmanovskoe forestry, $13 \mathrm{~km}$ NNE Ichalki, 4-20.VII.2017, 20.VII.1.VIII.2017, 3 ex., Semishin G.B.; Ardatov Distr.: Probuzhdenie, 2.VII.2008, 2 ex.; Dubenki Distr.: 8 km SE Engalychevo 1.VII.2009, 32 ex.; Lyambir Distr.: Atemar, 24.VI.2017, 1 ex.; Ruzaevka Distr.: Tatarskaya Pishlya, 30.VI.2008, 1 ex., Kurmaeva D.K.; Levzhensky, 21.VI.2009, 13.VI.2015, 2 ex.; Yakovshchina, 26.VI.2016, 1 ex.; Palaevka, 26.VI.2016, 1 ex.; Bolshie Berezniki Distr.: Gart, 19.VII.2008, 1 ex.; Simkino, 9.VII.2009, 1 ex.; Kochkurovo Distr.: Starye Turdaki, 8.VII.2008, 1 ex.; Muran, 29.VII.2017, 2 ex.; Mordovskoe Davydovo, 25.VI.2016, 1 ex.

DISTRIBUTION in Mordovia. Temnikov Distr. [Plavilshchikov, 1964; Ruchin, Kurmaeva, 2009; Orlov et al., 2011; Egorov, Ruchin, 2012, 2013a, 2014; Egorov et al., 2015 , 2016, 2017]; Zubova Polyana Distr. [Ruchin, Kurmaeva, 2009]; Ichalki Distr. [Ruchin, 2008a; Ruchin, Grishutkin, 2008]; Bolshie Berezniki Distr. [Timraleev, 1999; Ruchin, 2008a]. Saransk Town [Ruchin, 2009; Ruchin, Grishutkin, 2008]. Tengushevo Distr., Atyurjevo Distr., Elniki Distr., Krasnoslobodsk Distr., Staroe Shajgovo Distr., Ardatov Distr., Dubenki Distr., Lyambir Distr., Ruzaevka Distr., Kochkurovo Distr. [all this paper]

BIOLOGY in Mordovia. Habitats are similar to the previous species. Adult beetles visit inflorescences of Achillea millefolium L. s.1., Knautia arvensis (L.) Coult., Anthriscus sylvestris (L.) Hoffm., Angelica archangelica L., Angelica sylvestris L., Leucanthemum vulgare Lam., Aegopodium podagraria L., Filipendula ulmaria (L.) Maxim., Inula britannica L., Phleum pratense L., Plantago lanceolata L., Conium maculatum L., Cirsium arvense (L.) Scop., Calamagrostis arundinacea (L.) Roth, Daucus sativus (Hofmm.) Roehl., Fragaria vesca L., Tanacetum vulgare L., Erigeron canadensis L., Carduus acanthoides L., Cirsium arvense (L.) Scop. Imago flight lasts from the 1st decade of June to the 1st decade of August.

\section{Subfamilia Necydalinae}

\section{Necydalis major Linnaeus, 1758}

DISTRIBUTION in Mordovia. Temnikov Distr. [Egorov, Ruchin, 2013a, b; Ruchin et al., 2014; Ruchin, Egorov, 2015]. Ichalki Distr. [Ruchin, 2008a; Ruchin, Grishutkin, 2008; Ruchin, Egorov, 2015]; Chamzinka Distr. [Ruchin, Egorov, 2015]; Bolshie Berezniki Distr. [Timraleev, 1999; Ruchin, Egorov, 2015].

BIOLOGY in Mordovia. It is a rare species inhabiting on willow (Salix sp.) species.

\section{Subfamilia Spondylidinae}

\section{Asemum striatum (Linnaeus, 1758)}

MATERIAL. Temnikov Distr.: Veselyj, 25.V.2013, 2 ex.; Ardatov Distr.: 4,5 km SW Oktyabrsky, 19.V.2008, 2 ex.

DISTRIBUTION in Mordovia. Temnikov Distr. [Mozolevskaya et al., 1971; Egorov, Ruchin, 2013a, 2014; Egorov et al., 2015, 2016, 2017]; Zubova Polyana Distr. [Ruchin, 2008a].

BIOLOGY in Mordovia. It occurs in mixed forests, in ripe pine forests with spruce, birch, aspen. Sometimes it is caught next to freshly cut or hewn logs, on wooden poles of power lines. Imago flight lasts from the 2 nd decade of May to the 1 st decade of June.

\section{Arhopalus rusticus (Linnaeus, 1758)}

MATERIAL. Temnikov Distr.: Bochino, VII.2008, 1 ex., Susarev S.V.; Russkoe Karaevo, 20.VI.2015, 1 ex.; MSNR, Pushta, 56.VII.2017, 5 ex., Benediktov A.A.; $15 \mathrm{~km} \mathrm{~N} \mathrm{Temnikov,} \mathrm{26.VII.2017,}$ 1 ex.; 25 km E Temnikov, 3.VIII.2017, 2 ex.; same label, 6.VIII.2017, 2 ex.; 22 km N Temnikov, 4.VIII.2017, 1 ex., Semishin G.B.; 15 km N Temnikov, 6-24.VIII.2017, 2 ex., Egorov L.V., Semishin G.B.; Zubova Polyana Distr.: Vadovo-Sosnovka, 30.VII.2009, 1 ex.; Ichalki Distr.: NPS, Kemlyanskoe forestry, 9 km NE Ichalki, 15.VIII.2017, 4.VII.2017, 1.VIII.2017, 14.VIII.2017, 17.VIII.2017, 8 ex., Semishin G.B.; Bolshie Berezniki Distr.: 9 km S Simkino, 20.VI.2008, 16.VII.2009, 2 ex., Lobachev E.A.

DISTRIBUTION in Mordovia. Temnikov Distr. [Bondarenko, 1964; Plavilshchikov, 1964; Ruchin, Kurmaeva, 2009; Egorov, Ruchin, 2012; Egorov et al., 2015, 2016, 2017]; Ichalki Distr. [Ruchin, 2008a; Ruchin, Grishutkin, 2008]. Zubova Polyana Distr., Bolshie Berezniki Distr. [all this paper].

BIOLOGY in Mordovia. Inhabits mixed forests, pine forests with spruce, birch, ripe pine forests. It is often caught on rotten pine logs, inside wooden premises. Sometimes flies into the light. Imago flight lasts from the 2 nd decade of May to the 3rd decade of August.

\section{Tetropium castaneum (Linnaeus, 1758)}

MATERIAL. Temnikov Distr.: MSNR, 15 km N Temnikov, 115.VI.2017, 2 ex., Egorov L.V., Semishin G.B.

DISTRIBUTION in Mordovia. Temnikov Distr. [Mozolevskaya et al., 1971; Egorov, Ruchin, 2013a; Egorov et al., 2015, 2016, 2017]; Ichalki Distr. [Ruchin, 2008a; Ruchin, Grishutkin, 2008]; Bolshie Berezniki Distr. [Timraleev, 1999].

BIOLOGY in Mordovia. It occurs in pine forests with spruce, in ripe pine forests with spruce and birch, in spruce forests with pine, birch, in ripe spruce forests with pine, birch. Imago flight lasts from the 2 nd decade of May to the 1 st decade of August.

Tetropium fuscum (Fabricius, 1787)

DISTRIBUTION in Mordovia. Temnikov Distr. [Egorov et al., 2016].

BIOLOGY in Mordovia. In 2015 there were caught only 6 ex., in two quarters of Mordovia reserve. Habitats are ripe spruce forests with pine, birch and mixed forests.

\section{Spondylis buprestoides (Linnaeus, 1758)}

MATERIAL. Tengushevo Distr.: Khlebino, 2.VIII.2014, 1 ex.; Temnikov Distr.: Bochino, VII.2008, 2 ex., Susarev S.V.; Lavrentjevo, 6.VII.2013, 1 ex.; Sosnovka, 20.VII.2013, 1 ex.; Russkoe Karaevo, 20.VI.2015, 1 ex.; MSNR, Pushta, 5-6.VII.2017, 3 ex., Benediktov A.A.; 25 km E Temnikov, 6.VIII.2017, 1 ex., Semishin G.B.; 18 km NNE Temnikov, 24.VIII.2017, 1 ex.; Atyurjevo Distr.: Klopinka, 29.VI.2013, 1 ex.; Elniki Distr.: Novye Shaly, 19.VII.2015, 1 ex.; Kovylkino Distr.: Silikatny, 8.VIII.2013, 1 ex.; Ichalki Distr.: NPS, Barakhmanovskoe forestry, $13 \mathrm{~km}$ NNE Ichalki, 4-20.VII.2017, 1 ex., Semishin G.B.; NPS, Barakhmanovskoe forestry, 12 km NE Ichalki, 25.VII.2017, 1 ex.; NPS, Kemlyanskoe forestry, $9 \mathrm{~km}$ NE Ichalki, 31.VII.2017, 2.VIII.2017, 15.VIII.2017, 5 ex., Semishin G.B.; NPS, 15 km NNE Ichalki, 17.VII.2017, 1 ex., Semishin G.B.; Bolshie Berezniki Distr.: 5 km SE Permisi, 24.VII.2008, 1 ex., Kurmaeva D.K.

DISTRIBUTION in Mordovia. Temnikov Distr. [Bondarenko, 1964; Plavilshchikov, 1964; Ruchin, Kurmaeva, 2009; Egorov, Ruchin, 2012, 2013a, 2014; Egorov et al., 2015, 2016, 2017]; Zubova Polyana Distr. [Ruchin, Kurmaeva, 2009]; Ichalki Distr. [Ruchin, 2008a; Ruchin, Grishutkin, 2008]; Bolshie Berezniki Distr. [Timraleev, 1999; Ruchin, 2008a]. Tengushevo Distr., Atyurjevo Distr., Elniki Distr., Kovylkino Distr. [all this paper]. 
BIOLOGY in Mordovia. It occurs in pine forests of different types, spruce and mixed forests. Beetles can be found on wooden buildings, fallen trees, sawn timber. Flies into the light. Imago flight lasts from the 2 nd decade of June to the 1st decade of September.

\section{Subfamilia Cerambycinae}

Trichoferus campestris (Faldermann, 1835)

MATERIAL. Temnikov Distr.: MSNR, 25 km E Temnikov, 6.VIII.2017, 1 ex., Semishin G.B.; Kovylkino Distr.: Krasnaja Presnja, 31.VII.2012, 1 ex., Semishin G.B.; Ichalki Distr.: NPS, Kemlyanskoe forestry, $9 \mathrm{~km} \mathrm{NE}$ Ichalki, 27.VII.2017, 16.VIII.2017, 3 ex., Semishin G.B.

DISTRIBUTION in Mordovia. Saransk Town [Ruchin, 2008b, 2009; Semishin, 2009]. Temnikov Distr., Kovylkino Distr., Ichalki Distr. [all this paper].

BIOLOGY in Mordovia. Found in wooden buildings, in wooded areas.

REMARK. This species, alien to the region, has now spread in many regions of Russia, Middle Asia, in the last 20 years it has penetrated into different countries of western Europe, appeared from North America [Grebennikov et al., 2010; Dascãlu et al., 2013; Kadyrov et al., 2016; Pennacchio et al., 2016].

\section{Purpuricenus kaehleri (Linnaeus, 1758)}

DISTRIBUTION in Mordovia. Temnikov Distr. [Red Data Book ..., 2005; Ruchin, Egorov, 2015]; Chamzinka Distr. [Ruchin et al., 2014; Ruchin, Egorov, 2015]; Bolshie Berezniki Distr. [Timraleev, 1999; Red Data Book ..., 2005; Ruchin, Egorov, 2015]

BIOLOGY in Mordovia. Occurs sporadically in oak forests

\section{Aromia moschata (Linnaeus, 1758)}

MATERIAL. Temnikov Distr.: MSNR, 18 km NW Temnikov, 5.VIII.2017, 1 ex., Bolshakov L.V.; 25 km E Temnikov, 6.VIII.2017, 1 ex.; Elniki Distr.: Malye Mordovskie Poshaty, 22.VII.2017, 1 ex.; Kochkurovo Distr.: Muran, 29.VII.2017, 1 ex.

DISTRIBUTION in Mordovia. Tengushevo Distr. [Ruchin et al., 2014, 2015; Ruchin, Egorov, 2015]; Temnikov Distr. [Plavilshchikov, 1964; Ruchin et al., 2010, 2014 2015, 2017; Ruchin, Egorov, 2015]; Zubova Polyana Distr. [Ruchin, Kurmaeva, 2009; Ruchin, Egorov, 2015]; Elniki Distr. [Ruchin et al., 2017]; Krasnoslobodsk Distr. [Ruchin, Kurmaeva, 2009; Ruchin et al., 2010; Ruchin, Egorov, 2015]; Kovylkino Distr. [Ruchin, Egorov, 2015]; Staroe Shajgovo Distr. [Ruchin et al., 2010; Ruchin, Egorov, 2015]; Ichalki Distr. [Ruchin, 2008a; Ruchin, Grishutkin, 2008; Ruchin et al., 2010; Ruchin, Egorov, 2015]; Chamzinka Distr. [Ruchin et al., 2017]; Bolshie Berezniki Distr. [Timraleev, 1999; Ruchin, 2008a; Ruchin, Egorov, 2015]; Kochkurovo Distr. [Semishin, 2009; Ruchin et al., 2010; Ruchin, Egorov, 2015]. Saransk Town [Ruchin, 2008a, 2009; Ruchin, Egorov, 2015; Ruchin et al., 2016]. Elniki Distr. [this paper].

BIOLOGY in Mordovia. Single specimens usually occur in glades, fringes of mixed and deciduous forests, in floodplain meadows near forest tracts, in willows along river banks. Imago was observed on the inflorescences of Angelica sylvestris L., Filipendula ulmaria (L.) Maxim. Imago flight lasts from the 3rd decade of May to the 2nd decade of August.

*Obrium cantharinum (Linnaeus, 1767)

MATERIAL. Temnikov Distr.: MSNR, $15 \mathrm{~km} \mathrm{~N}$ Temnikov, 13-27.VII.2017, 1 ex., Egorov L.V., Semishin G.B.; Kochkurovo Distr.: Kochkurovo, VII.2009, 1 ex., Timoshkina L.; Saransk, 3.VI.2009, 1 ex.
DISTRIBUTION in Mordovia. Temnikov Distr., Kochkurovo Distr. [all this paper].

BIOLOGY in Mordovia. Single findings. In the city of Saransk, caught on the balcony of the house. In Mordovia Reserve, it is collected in a window trap in a linden-aspen hairy sedge forest with spruce. It is rather secretive species, its distribution is confined to the old aspen trees, in settlements it populates poplar plantations [Vlasov, 2015].

\section{*Deilus fugax (Olivier, 1790)}

MATERIAL. Temnikov Distr.: MSNR, $18 \mathrm{~km} \mathrm{~N}$ Temnikov, 26.V.2016, 1 ex.; MSNR, $20 \mathrm{~km} \mathrm{~N}$ Temnikov, 27.V.2016, 1 ex.

DISTRIBUTION in Mordovia. Temnikov Distr. [this paper]

BIOLOGY in Mordovia. According to some information [Adlbauer, 2001; Shapovalov, 2012]; it occurs in steppe biotopes, on heated xerophytic sites. We caught it in the forest quarters of Mordovia Reserve (coniferous and mixed forests). Trophically associated with Genista, Calycotome, Retama, Chamaecytisus, Cytisus [Navarro et al., 2005, Hidalgo-Fontiveros, 2010; Shapovalov, 2012]; in Mordovia, apparently with Genista tinctoria L. and Chamaecytisus ruthenicus (Fisch. Ex Woloszcz.). Flight takes place in May - June.

Molorchus minor (Linnaeus, 1758)

MATERIAL. Temnikov Distr.: Purdoshki, VI.2009, 1 ex., Susarev S.V.; Veselyj, 25.V.2013, 1 ex.; Temnikov Distr.: MSNR, 15 km N Temnikov, 12.V.-1.VI.2017, 1-15.VI.2017, 10 ex., Egorov L.V., Semishin G.B.; Ardatov Distr.: Olevka, 26.V.2017, 1 ex.; Saransk, 12.V.2009, 2 ex.

DISTRIBUTION in Mordovia. Temnikov Distr. [Feoktistov, 2011; Ruchin, Kurmaeva, 2009; Egorov, Ruchin, 2013a, 2014; Egorov et al., 2015, 2016, 2017]. Ardatov Distr., Saransk Town [all this paper].

BIOLOGY in Mordovia. It occurs along fringes, glades, in mixed forests, spruce forests with alder and birch, ripe pine forests with spruce and birch, sometimes in the gardens on flowering plants. Imago visit inflorescences of Sorbus aucuparia L., Aronia mitschurinii A. Skvorts. et Maitull., Padus avium Mill., Salix sp. Flight takes place in May-June.

* Molorchus marmottani Brisout de Barneville, 1863

MATERIAL. Temnikov Distr.: MSNR, 18 km N Temnikov, 15.VI.2016, 1 ex.

REMARK. Single specimens were collected for many years of research. In the Middle Volga region it is indicated in Chuvashia [Egorov, 2006]; Ulyanovsk [Isayev, Ishutov, 2001] and Samara regions [Isaev, Magdeev, 2003].

Ropalopus macropus (Germar, 1823)

DISTRIBUTION in Mordovia. Kochkurovo Distr. [Ruchin, 2008b].

BIOLOGY in Mordovia. There was caught single specimen only on the linden leaf. The only location in the republic is at the edge of deciduous forest of secondary origin

Semanotus undatus (Linnaeus, 1758)

DISTRIBUTION in Mordovia. Temnikov Distr. [Egorov et al., 2015].

BIOLOGY in Mordovia. The only one specimen was found in Mordovia reserve on a glade in a mixed forest. It inhabits coniferous forests, ecologically associated with spruce plantations. It uses shrunken and physiologically weakened trees, as well as spruce windbreaks [Cherepanov, 1981].

Callidium aeneum (DeGeer, 1775)

MATERIAL. Temnikov Distr.: Tretjakovo, 17.V.2014, 1 ex. 
DISTRIBUTION in Mordovia. Temnikov Distr. [Feoktistov, 2011; Egorov, Ruchin, 2013a; Egorov et al., 2015].

BIOLOGY in Mordovia. Single specimens are found in mixed and pine forests. It develops on deciduous and coniferous trees [Cherepanov, 1981].

Callidium coriaceum (Paykull, 1800)

MATERIAL. Ichalki Distr.: NPS, Lvovskoe forestry, $15 \mathrm{~km}$ NNE Ichalki, 4.VII.2017, 1 ex., Semishin G.B.

DISTRIBUTION in Mordovia. Temnikov Distr. [Egorov, Ruchin, 2014; Egorov et al., 2016]. Ichalki Distr. [this paper].

BIOLOGY in Mordovia. It was caught in a mixed forest, in ripe pine forest with a spruce, birch and in ripe spruce forest with a pine, birch. Larvae develop in the coniferous trees Picea, Abies, Pinus, preferring Larix [Doychev, Bencheva, 2008]. Imago flight lasts from the 2 nd decade of May to the 2 nd decade of July.

\section{Callidium violaceum (Linnaeus, 1758)}

MATERIAL. Temnikov Distr.: Bochino, VII.2008, 1 ex., Susarev S.V.; Ichalki Distr.: NPS, Lvovskoe forestry, $15 \mathrm{~km} \mathrm{NNE}$ Ichalki, 21.V.2008, 2 ex.; NPS, Kemlyanskoe forestry, 4 km NNE Ichalki, 23.V.2008, 2 ex.

DISTRIBUTION in Mordovia. Temnikov Distr. [Mozolevskaya et al., 1971; Egorov, Ruchin, 2013a, 2014; Egorov et al., 2015]; Zubova Polyana Distr. [Ruchin, 2008a]; Bolshie Berezniki Distr. [Timraleev, 1999]. Saransk Town [Semishin, 2009]. Ichalki Distr. [this paper].

BIOLOGY in Mordovia. It inhabits mixed and pine forests. Occurs in wooden buildings, on sawn timber. Imago flight lasts from the 2nd decade of May to the 1st decade of July.

Phymatodes abietinus Plavilstshikov et Lurie, 1960

DISTRIBUTION in Mordovia. Temnikov Distr. [Egorov, Shapovalov, 2017].

REMARK. It is known from some regions to the east of Mordovia [Egorov, Shapovalov, 2017]. Taking into account this finding, the range of the species can probably be considered European-West Siberian. Rare taiga species, leading a secretive way of life. According to the authors of all publications on the species, it develops on fir. Since in Mordovia, in the spot where $P h$. abietinus was found, conifers are presented by spruce and pine only, larvae develop, probably, on one of these tree species [Egorov et al., 2016].

\section{Plagionotus arcuatus (Linnaeus, 1758)}

MATERIAL. Temnikov Distr.: Veselyj, 25.V.2013, 1 ex.; Staroe Shajgovo Distr.: Staroe Akshino, 11.V.2008, 1 ex.; Chamzinka Distr.: Komsomolskij, VI.2008, VI.2009, 2 ex., Ryzhov M.K.

DISTRIBUTION in Mordovia. Temnikov Distr. [Feoktistov, 2011; Ruchin, Kurmaeva, 2009]; Zubova Polyana Distr., Chamzinka Distr. [Ruchin, 2008a]; Bolshie Berezniki Distr. [Timraleev, 1999]. Staroe Shajgovo Distr. [this paper]

BIOLOGY in Mordovia. It occurs in broadleaf and mixed forests. Mating species are found on the trunk of a fallen oak tree, on oak logs. Larvae develop under the bark of deciduous trees, preferring Quercus [Shapovalov, 2012]. Imago flight lasts from the 2nd decade of May to the 1st decade of June.

Plagionotus detritus (Linnaeus, 1758)

DISTRIBUTION in Mordovia. Temnikov Distr. [Feoktistov, 2011; Ruchin, Kurmaeva, 2009]; Ichalki Distr. [Ruchin, Egorov, 2013]

BIOLOGY in Mordovia. It occurs in the same biotopes as the previous species. The remains of individuals were found under the bark of oak.

\section{Chlorophorus herbstii (Brahm, 1790)}

MATERIAL. Staroe Shajgovo Distr.: Lemdyajsky Majdan, VI.2008, 1 ex., Malygina T.; Lesnichestvo, 17.VII.2009, 2 ex.; Ardatov Distr.: Kozlovka, 2007, 2 ex.; Kochkurovo Distr.: Starye Turdaki, 13.VII.2008, 1 ex.; Bolshie Berezniki Distr.: Simkinskoe lesnichestvo, 12.VIII.2017, 1 ex.

DISTRIBUTION in Mordovia. Temnikov Distr. [Ruchin, Kurmaeva, 2009; Egorov, Ruchin, 2013a, 2014]; Chamzinka Distr. [Ruchin, 2008a]; Kochkurovo Distr. [Semishin, 2009]. Staroe Shajgovo Distr., Ardatov Distr., Bolshie Berezniki Distr. [all this paper].

BIOLOGY in Mordovia. It occurs in mixed, broad-leaved forests, in floodplain meadows. Imago visit inflorescences of Angelica sylvestris L., Rubus sp., Aegopodium podagraria L. Imago flight lasts from the 1st decade of June to the 2nd of August.

? Chlorophorus varius (O.F. Müller, 1766)

DISTRIBUTION in Mordovia. Temnikov Distr. [Feoktistov, 2011].

BIOLOGY in Mordovia. It is indicated only from the territory of Mordovia Reserve. Larvae develop under the bark and in the wood of shrunken deciduous trees and shrubs (Chamaecytisus, Eleagnus, Acer, Populus, Quercus, Ulmus, Tilia, Malus, Prunus, Caragana), as well as on Salicornia, Achillea [Bartenev, 2004; Shapovalov, 2012].

*Rhaphuma gracilipes (Faldermann, 1835)

MATERIAL. Temnikov Distr.: MSNR, $32 \mathrm{~km} \mathrm{C} \mathrm{Temnikov,}$ 18.VI.2016, $1 \mathrm{ex}$

REMARK. The only finding in Mordovia reserve was made in the quarter 37. Palaearctic species, quite common in the eastern part of the range. In the Middle Volga region it is indicated from Chuvashia [Egorov, 2009]; Mariy-El [Kurzawa, 2012] and Samara region [Isajev et al., 2004].

Xylotrechus capricornis Gebler, 1830

DISTRIBUTION in Mordovia. Temnikov Distr. [Egorov, Ruchin, 2013a].

BIOLOGY in Mordovia. The only find (3 specimens) was made in a mixed forest. It is considered a monophage on Betula sp. [Sláma, Gutowski, 1997]. Larvae develop in the upper parts of trunks of weakened or recently dead trees.

\section{Xylotrechus rusticus (Linnaeus, 1758)}

MATERIAL. Temnikov Distr.: Novye Shaly, VII.2009, 1 ex., Susarev S.V.; Veselyj, 25.V.2013, 4 ex.; MSNR, 15 km N Temnikov, 2.VI.2017, 1 ex., Semishin G.B.; 31 km NE Temnikov, 13.VII. 2017, 1 ex.; Staroe Shajgovo Distr.: Staroe Akshino, 11.V.2008, 1 ex.; Ichalki Distr.: NPS, Lvovskoe forestry, $15 \mathrm{~km}$ NNE Ichalki, 21.V.2008, 3 ex.; Ardatov Distr.: Oktyabrsky, 19.V.2008, 1 ex.; Lyambir Distr.: Ekaterinovka, 2.VI.2009, 5 ex.; Bolshie Berezniki Distr.: 9 km S Simkino, 20.VI.2008, 1 ex., Lobachev E.A.

DISTRIBUTION in Mordovia. Temnikov Distr. [Redikortsev, 1938; Plavilshchikov, 1964; Mozolevskaya et al., 1971; Ruchin, Kurmaeva, 2009; Egorov, Ruchin, 2012, 2013a, 2014; Egorov et al., 2015, 2016, 2017]; Ichalki Distr. [Ruchin, 2008a; Ruchin, Grishutkin, 2008]; Bolshie Berezniki Distr. [Timraleev, 1999]. Staroe Shajgovo Distr., Ardatov Distr., Lyambir Distr. [all this paper].

BIOLOGY in Mordovia. Occurs in deciduous and mixed forests. It was observed on the trunk of the Betula sp., birch logs, but it was more often noticed on aspen logs, dying or fallen aspen trees. Imago flight lasts from the 2 nd decade of May to the 2nd decade of July. 


\section{Cyrtoclytus capra (Germar, 1823)}

DISTRIBUTION in Mordovia. Temnikov Distr. [Egorov, Ruchin, 2013a; Egorov et al., 2016, 2017]; Bolshie Berezniki Distr. [Ruchin et al., 2009].

BIOLOGY in Mordovia. Single specimens were registered in deciduous forests and on the edge of the pine forest with spruce, birch, alder. Imago were found on the inflorescences of plants from the Umbelliferae family. Imago flight lasts from the 2 nd decade of June to the 1 st decade of July.

*Clytus arietis (Linnaeus, 1758)

MATERIAL. Temnikov Distr.: MSNR, 15 km N Temnikov, 12. V.2016, 2 ex., Egorov L.V.; 21.V.2016, 2 ex.; 18 km NW Temnikov, 13.VI.2016, 2 ex.; Lyambir Distr.: Ekaterinovka, 2.VI. 2009, 1 ex.

DISTRIBUTION in Mordovia. Temnikov Distr., Lyambir Distr. [all this paper].

BIOLOGY in Mordovia. It was found on the edge of deciduous forest, in floodplain deciduous forests (site with alder and birch), on a glade in the mixed forest on the leaf of Rubus idaeus L. Larvae develop under the bark and in the wood of dead trunks and shoots of various deciduous trees and shrubs, including Quercus, Ulmus, Betula, Tilia, Salix, Acer, Rosa (and other Rosaceae) [Shapovalov, 2012; Georgiev et al., 2015]. Imago flight lasts from the 3rd decade of May to the 2nd decade of June.

\section{Subfamilia Lamiinae \\ Mesosa myops (Dalman, 1817)}

MATERIAL. Tengushevo Distr.: Barashevo, 30.VI.2013, 1 ex.; Temnikov Distr.: Andreevka, 11.V.2013, 1 ex.; MSNR, 22 km N Temnikov, 20.V.2017, 1 ex.; Atyurjevo Distr.: Mordovskaya Kozlovka, 10.VIII.2014, 1 ex.; Chamzinka Distr.: Komsomolskij, 18.V.2008, 1 ex., Ryzhov M.K.; Bolshie Berezniki Distr.: 9 km S Simkino, 29.VI.2009, 1 ex.

DISTRIBUTION in Mordovia. Temnikov Distr. [Egorov, Ruchin, 2013a; Egorov et al., 2016, 2017]; Kovylkino Distr. [Ruchin, 2008a]; Ichalki Distr. [Ruchin, 2008a; Ruchin, Grishutkin, 2008]. Saransk Town [Ruchin, 2009]. Tengushevo Distr., Atyurjevo Distr., Chamzinka Distr., Bolshie Berezniki Distr. [all this paper].

BIOLOGY in Mordovia. It occurs in mixed and deciduous forests, in pine forests with an admixture of birch. Often can be seen in towns and cities. We observed it on the wall of a wooden house, on an old oak tree, on a trunk of an apple tree, on a birch log. Imago flight lasts from the $3 \mathrm{rd}$ decade of April to the 1st decade of August.

\section{Monochamus galloprovincialis (Olivier, 1795)}

In Mordovia there is a subspecies M. galloprovincialis pistor (Germar, 1818).

MATERIAL. Tengushevo Distr.: Khlebino, 18.VII.2015, 1 ex. Temnikov Distr.: Russkoe Karaevo, 20.VI.2015, 1 ex.; Veselyj, 6.VI.2015, 1 ex.; Bochino, VII.2008, 1 ex., Susarev S.V.; Zubova Polyana Distr.: Zubova Polyana, 2.VIII.2008, 2 ex., Kurmaeva D.K.; Zhukovka, 31.VII.2009, 5 ex.; Elniki Distr.: Novye Shaly, 19. VII.2015, 1 ex.; Ichalki Distr.: Sosnovka, 6.VIII.2008, 2 ex.; NPS, Barakhmanovskoe forestry, $13 \mathrm{~km}$ NNE Ichalki, 6.IX.2009, 5 ex.; NPS, Barakhmanovskoe forestry, 11 km NE Ichalki, 6.IX.2009, 2 ex.; NPS, Barakhmanovskoe forestry, $16 \mathrm{~km}$ NEE Ichalki, 11.IX.2017, 1 ex., Semishin G.B.; Ardatov Distr.: 3 km NW Sosnovoe, 18.VIII.2008, $10^{7}, 1$, in copula; Chamzinka Distr.: Kosogory, 21.VIII.2008, 1 ex., Kurmaeva D.K.; Dubenki Distr.: Krasnye Luga, 11.VI.2017, 1 ex.; Bolshie Berezniki Distr.: 5 km SE Permisi, 24. VII.2008, 1 ex., Kurmaeva D.K.

DISTRIBUTION in Mordovia. Temnikov Distr. [Bondarenko, 1964; Plavilshchikov, 1964; Ruchin, Kurmaeva, 2009;
Egorov, Ruchin, 2012, 2013a, 2014; Egorov et al., 2015, 2016, 2017]; Ichalki Distr. [Ruchin, 2008a; Ruchin, Grishutkin, 2008]; Bolshie Berezniki Distr. [Ruchin, 2008a]. Tengushevo Distr., Zubova Polyana Distr., Elniki Distr., Chamzinka Distr., Dubenki Distr. [all this paper].

BIOLOGY in Mordovia. It was especially numerous in Mordovia reserve after the fires of 2010. In 2011-2013 its number burnt-out places, and on fallen pines on unburnt areas, located near the burnings, reached 150-200 ex., for a day excursion. At present, the population has stabilized to the level of 2009. Common habitats are mixed forests, coniferous forests of various types. It is very often found on pine $\operatorname{logs}$, on sawn timber, on logs peeled from bark, on fallen pine trees, on fresh wooden buildings. Imago flight lasts from the 3rd decade of May to the 2nd decade of September.

\section{Monochamus saltuarius (Gebler, 1830)}

DISTRIBUTION in Mordovia. Temnikov Distr. [Plavilshchikov, 1964; Mozolevskaya et al., 1971].

REMARK. The only indication in the republic was from the territory of Mordovia reserve. There are no recent finds. Representative of the taiga fauna.

\section{Monochamus sutor (Linnaeus, 1758)}

MATERIAL. Temnikov Distr.: Veselyj, 25.V.2013, 2 ex.; MSNR, Pushta, 5-6.VII.2017, 1 ex., Benediktov A.A.; $25 \mathrm{~km} \mathrm{E}$ Temnikov, 18.VI.2017, 1 ex.; 22 km N Temnikov, 4.VIII.2017, 1 ex., Semishin G.B.; Atyurjevo Distr.: Mordovskaya Kozlovka, 8.VI. 2013, 1 ex.; Ichalki Distr.: NPS, Lvovskoe forestry, 15 km NNE Ichalki, 17.VI.2008, 1 ex.

DISTRIBUTION in Mordovia. Temnikov Distr. [Mozolevskaya et al., 1971; Egorov, Ruchin, 2013a, 2014; Egorov et al., 2016, 2017]; Ichalki Distr. [Ruchin, 2008a; Ruchin, Grishutkin, 2008]; Bolshie Berezniki Distr. [Ruchin, 2008a]. Atyurjevo Distr. [this paper].

BIOLOGY in Mordovia. The number is lower than that of M. galloprovincialis pistor, but they are usually found in similar biotopes. Imago flight lasts from the $3 \mathrm{rd}$ decade of May to the 1st decade of August.

\section{Monochamus urussovii (Fischer von Waldheim,} 1805)

DISTRIBUTION in Mordovia. Temnikov Distr. [Plavilshchikov, 1964; Ruchin, Kurmaeva, 2009; Egorov, Ruchin, 2012, 2013a, 2014; Egorov et al., 2015, 2016].

BIOLOGY in Mordovia. Occurs less frequently than the previous species of the genus, but in similar biotopes. Imago flight lasts from the first decade of June to the 2nd decade of August.

\section{Lamia textor (Linnaeus, 1758)}

MATERIAL. Temnikov Distr.: Mikhajlovka, VI-VII.2012, 1 ex.; Bolshie Berezniki Distr.: 9 km S Simkino, 5.VIII.2013, 1 ex.

DISTRIBUTION in Mordovia. Temnikov Distr. [Redikortsev, 1938; Plavilshchikov, 1964; Ruchin, Kurmaeva, 2009; Ruchin et al., 2010, 2015]; Zubova Polyana Distr. [Ruchin, Kurmaeva, 2009; Ruchin et al., 2010]; Elniki Distr. [Ruchin et al., 2015]; Kovylkino Distr. [Ruchin et al., 2016]; Ichalki, Ardatov Distr. [Ruchin et al., 2010]; Bolshie Berezniki Distr. [Ruchin, 2008a; Ruchin et al., 2015].

BIOLOGY in Mordovia. Usually there are single individuals on the leaves of plants and shrubs. Rare species. The main habitats are mixed forests, wet aspen trees, willow trees along water bodies, wetlands. Imago flight lasts from the first decade of May to the 1st decade of August. 
Anaesthetis testacea (Fabricius, 1781)

DISTRIBUTION in Mordovia. Kochkurovo Distr. [Ruchin, 2008b].

BIOLOGY in Mordovia. Reliably known for 1 specimen, collected on the edge of deciduous forest of secondary origin. Larvae develop in shrunken and shrunking branches and thin trunks of Salix, Quercus, Alnus, Betula, Populus, Prunus, Rosa, Malus, Pyrus, Rubus, Cerasus, Ulmus, Tilia, Eleagnus [Foit, 2010; Shapovalov, 2012].

\section{Pogonocherus decoratus Fairmaire, 1855}

DISTRIBUTION in Mordovia. Temnikov Distr. [Feoktistov, 2011; Ruchin, Kurmaeva, 2009].

REMARK. The only find dates back to 1984 (Walsensky cordon, Mordovia Reserve). The main habitats are coniferous forests. Larvae develop under the bark and in the upper layers of wood in thin branches of predominantly coniferous species [Shapovalov, 2012].

\section{*Pogonocherus hispidulus \\ (Piller et Mitterpacher, 1783)}

MATERIAL. Temnikov Distr.: MSNR, 16 km N Temnikov, IV.2015, 1 eX.

BIOLOGY in Mordovia. There was only 1 ex. found in a coniferous-deciduous forest. Larvae develop under the bark of dead branches and thin trunks of various species of deciduous hardwood and shrubbs [Shapovalov, 2012].

\section{Pogonocherus fasciculatus (DeGeer, 1775)}

MATERIAL. Temnikov Distr.: MSNR, 20 km NNE Temnikov, VI-VII.2012, 1 ex.; Ichalki Distr.: NPS, Barakhmanovskoe forestry, $13 \mathrm{~km}$ NNE Ichalki, 1.V.2017, 1 ex.; NPS, Lvovskoe forestry, $15 \mathrm{~km}$ NNE Ichalki, 5-19.VI.2017, 1 ex., Semishin G.B.; Ardatov Distr.: Turgenevo, 19.V.2008, 1 ex.

DISTRIBUTION in Mordovia. Temnikov Distr. [Bondarenko, 1964; Plavilshchikov, 1964; Ruchin, Kurmaeva, 2009; Egorov, Ruchin, 2013a, 2014; Egorov et al., 2015, 2016, 2017]; Ichalki Distr. [Ruchin, Egorov, 2013]. Ardatov Distr. [this paper].

BIOLOGY in Mordovia. It is often observed on pine logs, fallen pines, on pine wooden $\log$ houses. Habitats are very diverse with the presence of pine: ripe pine forests with spruce, birch, aspen, fringes of pine forests with spruce, bog moss ripe pine forest with spruce, birch, pine forests with birch, mixed forests. Imago flight lasts from the 1st decade of May to the 1st decade of July.

\section{Aegomorphus clavipes (Schrank, 1781)}

MATERIAL. Staroe Shajgovo Distr.: Lesnichestvo, 17.VII.2009, 3 ex.; Lyambir Distr.: Ekaterinovka, 2.VI.2009, 2 ex.; Bolshie Berezniki Distr.: 9 km S Simkino, 2.VII.2009, 1 ex., Lobachev E.A.; Saransk, 8.VII.2012, 1 ex., Semishin G.B.

DISTRIBUTION in Mordovia. Temnikov Distr. [Feoktistov, 2011; Ruchin, Kurmaeva, 2009; Egorov, Ruchin, 2012, 2013a; Egorov et al., 2015, 2016, 2017]. Bolshie Berezniki Distr. [Timraleev, 1999]. Staroe Shajgovo Distr., Lyambir Distr., Saransk Town [all this paper].

BIOLOGY in Mordovia. It occurs in mixed and deciduous forests. Often caught on dry aspen, birch and aspen logs. Imago flight lasts from the 3rd decade of May to the 2nd decade of July.

Aegomorphus obscurior (Pic, 1904)

DISTRIBUTION in Mordovia. Temnikov Distr. [Egorov, Ruchin, 2013a].

BIOLOGY in Mordovia. The only specimen was caught in the vicinity of cordon Novenkij on birch firewood. Larvae develop in hardwood, more often in birch [Plewa et al., 2011].

$$
\text { Oplosia cinerea (Mulsant, 1839) }
$$

DISTRIBUTION in Mordovia. Kochkurovo Distr. [Ruchin, 2008b].

BIOLOGY in the Mordovia. The only location in the republic is at the edge of deciduous forest of secondary origin. Larvae develop under the bark and in lime wood [Shapovalov, 2012].

\section{Acanthocinus aedilis (Linnaeus, 1758)}

MATERIAL. Temnikov Distr.: Bochino, VI.2008, 1 ex., Susarev S.V.; Tretjakovo, 17.V.2014, 1 ex.; MSNR, Pushta, 5-6.VII. 2017, 1 ex., Benediktov A.A.; Veselyj, 25.V.2013, 2 ex.; Torbeevo Distr.: Vindrej, 6.VI.2008, 1 ex.; Atyurjevo Distr.: Stepanovka, 24.V.2014, 1 ex.; Ichalki Distr.: NPS, Kemlyanskoe forestry, $4 \mathrm{~km}$ NNE Ichalki, 18.IV.2009, 1 ex.; NPS, Kemlyanskoe forestry, 7 km NE Smolny, VII.2009, 1 ex.; Ardatov Distr.: Kelvjadni, 11.V.2009, 1 ex., Lukijanov S.V.; Oktyabrsky, 19.V.2008, 26.V.2017, $20^{\top}, 3$ ? Dubenki Distr.: Nikolaevka, 9.V.2007, 1 ex.; 5.IV.2008, $10^{7}$, 1 , in copula; Krasnye Luga, 11.VI.2017, 1 ex.; 8 km SE Engalychevo, 1.VII.2009, 1 ex.; Bolshie Berezniki Distr.: 6 km SE Permisi, 12.VI. 2015, 1 ex

DISTRIBUTION in Mordovia. Temnikov Distr. [Bondarenko, 1964; Plavilshchikov, 1964; Kirsta, 1974; Ruchin, 2008a; Ruchin, Kurmaeva, 2009; Egorov, Ruchin, 2012, 2013a, 2014; Egorov et al., 2015, 2016]; Zubova Polyana Distr. [Ruchin, 2008a]; Torbeevo Distr. [Ruchin, Kurmaeva, 2009]; Ichalki Distr. [Ruchin, 2008a; Ruchin, Grishutkin, 2008]; Dubenki, Bolshie Berezniki Distr. [Ruchin, 2008a]. Atyurjevo Distr., Ardatov Distr. [all this paper].

BIOLOGY in Mordovia. The habitats are mixed forests, pine and spruce forests of various types, clearings. It is often observed on pine logs, fallen pine trees, on pine wooden log houses. In Mordovia reserve, larvae were found under the bark of pine trees. Usually found in the lower part of the trunk at an altitude of $4 \mathrm{~m}$ [Bondarenko, 1964]. Imago flight lasts from the 3rd decade of April to the 1st decade of July

Acanthocinus griseus (Fabricius, 1792)

DISTRIBUTION in Mordovia. Temnikov Distr. [Mozolevskaya et al., 1971; Egorov, Ruchin, 2013a, 2014].

BIOLOGY in the Mordovia. The number is lower than that of the previous species. It occurs in the same biotops. Flies into the light.

\section{Leiopus linnei Wallin et al., 2009}

MATERIAL. Temnikov Distr.: MSNR, $15 \mathrm{~km} \mathrm{~N}$ Temnikov, 29.VI.-13.VII.2017, 1 ex., Egorov L.V., Semishin G.B.

DISTRIBUTION in Mordovia. Temnikov Distr. [Feoktistov, 2011; Ruchin, Kurmaeva, 2009]; Torbeevo, Lyambir Distr. [Ruchin, 2008b].

BIOLOGY in Mordovia. It occurs in mixed forests. Imago flight lasts from the 3 rd decade of May to the 2nd decade of July.

\section{Exocentrus lusitanus (Linnaeus, 1767)}

MATERIAL. Ichalki Distr.: NPS, Kemlyanskoe forestry, $9 \mathrm{~km}$ NE Ichalki, 15.VIII.2017, 1 ex., Semishin G.B.

DISTRIBUTION in Mordovia. Temnikov Distr. [Egorov, Ruchin, 2014; Egorov et al., 2015, 2016, 2017]. Ichalki Distr. [this paper].

BIOLOGY in Mordovia. It occurs in mixed forests, and was also registered in the floodplain deciduous forest with predominance of aspen. Flies into the light. 
Tetrops praeustus (Linnaeus, 1758)

MATERIAL. Temnikov Distr.: MSNR, 15 km N Temnikov, 115.VI.2017, 1 ex., Egorov L.V., Semishin G.B.

DISTRIBUTION in Mordovia. Temnikov Distr. [Egorov, Ruchin, 2012, 2013a, 2014; Egorov et al., 2017]; Chamzinka Distr. [Ruchin et al., 2009].

BIOLOGY in Mordovia. Common. Inhabits pine forests with birch, aspen, linden, spruce and in pine forests with spruce, birch, sometimes in moistened places. Often caught into the hoop-net when mowing Padus, Salix, Sorbus. Imago flight lasts from the 1 st decade of May to the 2nd decade of June.

Saperda carcharias (Linnaeus, 1758)

MATERIAL. Ichalki Distr.: NPS, Barakhmanovskoe forestry, 16 km NEE Ichalki, 13.IX.2017, 1 ex., Semishin G.B.; Bolshie Berezniki Distr.: 9 km S Simkino, 18.VII.2009, 1 ex., Lobachev E.A.

DISTRIBUTION in Mordovia. Temnikov Distr. [Plavilshchikov, 1964]; Ichalki Distr. [Ruchin, 2008a; Ruchin, Grishutkin, 2008]. Bolshie Berezniki Distr. [this paper].

BIOLOGY in Mordovia. It occurs in mixed and floodplain deciduous forests. Flies into the light.

Saperda perforata (Pallas, 1773)

MATERIAL. Temnikov Distr.: MSNR, 15 km N Temnikov, 2.VI.2017, 1 ex., Semishin G.B.; Bolshie Berezniki Distr.: 9 km S Simkino, 20.VI.2008, 1 ex., Lobachev E.A.

DISTRIBUTION in Mordovia. Temnikov Distr. [Feoktistov, 2011; Ruchin, Kurmaeva, 2009; Egorov, Ruchin, 2012; Egorov et al., 2015, 2016]. Saransk Town [Semishin, 2009]. Bolshie Berezniki Distr. [this paper].

BIOLOGY in Mordovia. It occurs in mixed forests, in ripe spruce forests with pine, birch, in aspen groves, willow thickets. Often observed on aspen logs. Imago flight takes place in June.

\section{Saperda populnea (Linnaeus, 1758)}

DISTRIBUTION in Mordovia. Ichalki Distr., Ruzaevka Distr. [Ruchin, 2008a; Ruchin, Grishutkin, 2008]; Bolshie Berezniki Distr. [Timraleev, 1999]. forests.

BIOLOGY in Mordovia. Occurs in mixed and deciduous

\section{Saperda scalaris (Linnaeus, 1758)}

MATERIAL. Kochkurovo Distr.: Kochkurovo, VII.2009, 1 ex. Timoshkina L.; Saransk Town, 7.VI.2012, 1 ex., Semishin G.B.

DISTRIBUTION in Mordovia. Temnikov Distr. [Mozolevskaya et al., 1971; Egorov, Ruchin, 2013a; Egorov et al., 2015, 2016, 2017]; Ichalki Distr. [Ruchin, 2008a; Ruchin, Grishutkin, 2008]; Chamzinka Distr. [Ruchin, 2008a]. Kochkurovo Distr., Saransk Town [all this paper].

BIOLOGY in Mordovia. It is noticed in mixed forests, pine forests with spruce, birch, rare young oaks, floodplain oak forests. Imago flight lasts from the 3rd decade of May to the 2 nd decade of July.

Stenostola dubia (Laicharting, 1784)

DISTRIBUTION in Mordovia. Temnikov Distr. [Egorov et al., 2015].

REMARK. The only specimen was collected in Mordovia reserve. In European part of Russia indications from the north and center are extremely rare. Reported from Leningrad, Vladimir and Orenburg regions [Shapovalov, 2012].

Stenostola ferrea (Schrank, 1776)

MATERIAL. Temnikov Distr.: MSNR, 18 km NNE Temnikov, V-VI.2012, 1 ex.
DISTRIBUTION in Mordovia. Temnikov Distr. [Feoktistov, 2011; Ruchin, Kurmaeva, 2009].

BIOLOGY in Mordovia. Sporadically occurs in mixed forests. Larvae develop under the bark of branches and tree trunks of hardwood trees [Shapovalov, 2012].

Oberea oculata (Linnaeus, 1758)

MATERIAL. Temnikov Distr.: MSNR, Pushta, 9.V.2009, 1 ex.; 25 km E Temnikov, 24.VI.2016, 1 ex., Egorov L.V. Elniki Distr.: Bolshoj Urkat, 13.V.2009, 2 ex.

DISTRIBUTION in Mordovia. Chamzinka Distr. [Ryzhov, 2012]. Temnikov Distr., Elniki Distr. [all this paper].

BIOLOGY in Mordovia. Rare species. It was caught on the floodplain meadows in willow thickets, in mixed forests, at the edge of pine forest with birch on the shoots of Salix caprea $\mathrm{L}$.

Phytoecia coerulescens (Scopoli, 1763)

MATERIAL. Ichalki Distr.: NPS, Lvovskoe forestry, $15 \mathrm{~km} \mathrm{NNE}$ Ichalki, VII.2008, 1 ex., Lobachev E.A.; Saransk, 2.IV.2013, 1 ex.

DISTRIBUTION in Mordovia. Temnikov Distr. [Egorov, Ruchin, 2013a]. Zubova Polyana Distr. [Ruchin, 2008b; Ruchin, Kurmaeva, 2009]. Ichalki Distr., Saransk Town [all this paper].

BIOLOGY in Mordovia. Registered on the fringes and glades of the mixed forests, meadows. Larvae develop on plants of the families Boraginaceae (Lithospermum, Echium, Anchusa, Lycopsis, Lappula, Cynoglossum, Symphytum, Cerinthe), Labiatae (Salvia), Asteraceae (Inula) [Coleopterous insects..., 2010].

\section{Phytoecia cylindrica (Linnaeus, 1758)}

MATERIAL. Temnikov Distr.: MSNR, 18 km NW Temnikov, 20.V.2017, 1 ex.; $15 \mathrm{~km} \mathrm{~N}$ Temnikov, 15.VI.2017, 1 ex.; $25 \mathrm{~km} \mathrm{E}$ Temnikov, 16.VI.2017, 1 ex., Semishin G.B.; Krasnoslobodsk Distr.: 2 km N Selishchi, 5.VI.2009, 1 ex.; Ichalki Distr.: NPS, Lvovskoe forestry, 15 km NNE Ichalki, 23.V.2017, 1 ex., Semishin G.B.; Insar Distr.: Kochetovka, 4.VI.2016, 5 ex.; Chamzinka Distr.: Bolshoe Maresevo, 13.VI.2008, 2 ex.; Komsomolskij, 30.V.2009, 1 ex., Ryzhov M.K.; Lyambir Distr.: Ekaterinovka, 29.V.2008, 2.VI.2009, 2 ex.; Ruzaevka Distr.: Levzhensky, 9.VI.2009, 1 ex.; 3 km N Streletskaya Sloboda, 20.VI.2009, 1 ex.; Bolshie Berezniki Distr.: Simkino, 9.VII.2009, 1 ex.; Saransk, 31.V.2009, 2 ex.

DISTRIBUTION in Mordovia. Temnikov Distr. [Ruchin, 2008b; Ruchin, Kurmaeva, 2009; Egorov, Ruchin, 2012, 2013a, 2014; Egorov et al., 2015, 2016, 2017]; Torbeevo Distr. [Ruchin, 2008b; Ruchin, Kurmaeva, 2009]; Ichalki Distr., Ardatov Distr., Lyambir Distr. [Ruchin, 2008b]. Krasnoslobodsk Distr., Chamzinka Distr., Ruzaevka Distr., Bolshie Berezniki Distr., Saransk Town [this paper].

BIOLOGY in Mordovia. It occurs along the fringes of mixed and deciduous forests, in floodplain deciduous forests, in linden groves with aspen, pine and spruce. It is often found on herbaceous plants. Imago flight lasts from the 2 nd decade of May to the 1st decade of August

*Phytoecia icterica (Schaller, 1783)

MATERIAL. Ruzaevka Distr.: Levzhensky, 9.VI.2009, 1 ex. DISTRIBUTION in Mordovia. Ruzaevka Distr. [this paper].

BIOLOGY in Mordovia. The only specimen was caught at the edge of a deciduous forest along the border with a stepped slope.

Phytoecia nigricornis (Fabricius, 1781)

MATERIAL. Oktyabrsky Distr.: Monastyrskoe, 12.VI.2017, 1 ex. DISTRIBUTION in the Mordovia. Temnikov Distr. [Egorov et al., 2016, 2017]; Oktyabrsky Distr. [this paper]. 
BIOLOGY in Mordovia. Found on the edge of a mixed forest, caught on Artemisia absinthium L.

Phytoecia pustulata (Schrank 1776)

MATERIAL. Elniki Distr.: Starye Shaly, V-VI.2014, 1 ex. DISTRIBUTION in Mordovia. Temnikov Distr. [Egorov, Ruchin, 2012; Egorov et al., 2016, 2017]; Elniki Distr. [this paper].

BIOLOGY in Mordovia. The habitats are pine forests with birch, aspen, linden, spruce, ripe spruce forest with pine, birch, with well developed grass cover.

Agapanthia cardui (Linnaeus, 1767)

MATERIAL. Temnikov Distr.: Veselyj, 6.VI.2015, 1 ex.; MSNR, 18 km NW Temnikov, 3.VI.2017, 15.VII.2017, 2 ex.; 22 km N Temnikov, 4.VII.2017, 1 ex.

DISTRIBUTION in Mordovia. Temnikov Distr. [Egorov, Ruchin, 2014; Egorov et al., 2015, 2016, 2017]; Krasnoslobodsk Distr. [Ruchin et al., 2009].

BIOLOGY in Mordovia. It occurs along the fringes of ripe pine forests with spruce, birch, aspen, ripe pines with spruce, deciduous forests. Once mown off with Cyperaceae in a damp lowland on a burner. Imago flight lasts from the $3 \mathrm{rd}$ decade of May to the 2nd decade of July.

\section{Agapanthia intermedia Ganglbauer, 1884}

MATERIAL. Temnikov Distr.: MSNR, 18 km NNE Temnikov, 18.VI.2017, 1 ex.; $15 \mathrm{~km} \mathrm{~N}$ Temnikov, 29.VI.2017, 1 ex., Semishin G.B.; Krasnoslobodsk Distr.: Krasnoslobodsk, 5.VI.2009, 3 ex.; Ichalki Distr.: NPS, Lvovskoe forestry, $15 \mathrm{~km}$ NNE Ichalki, 30.VI 2008, 1 ex.; Khanineevka, 12.VI.2009, 1 ex.

DISTRIBUTION in Mordovia. Temnikov Distr. [Egorov, Ruchin, 2012, 2013a; Egorov et al., 2016, 2017]. Saransk Town [Ruchin, 2008a, 2009]. Krasnoslobodsk Distr., Ichalki Distr. [all this paper].

BIOLOGY in Mordovia. Occurs in birch forests with pine, aspen, linden, along the fringes of deciduous forests of secondary origin, in mixed forests. Imago noticed on Knautia arvensis (L.) Coult. Monophagus larvae - on Knautia. Imago flight lasts from the 3rd decade of May to the 1st decade of July.

Agapanthia villosoviridescens (DeGeer, 1775)

MATERIAL. Temnikov Distr.: MSNR, 18 km N Temnikov, VI-VII.2012, 1 ex.; Atyurjevo Distr.: Stepanovka, 24.V.2014, 1 ex.; Elniki Distr.: Novye Shaly, 26.V.2013, 1 ex.; Krasnoslobodsk Distr.: Krasnoslobodsk, 5.VI.2009, 1 ex.; 2 km N Selishchi, 5.VI.2009, 5 ex.; Bolshoe Ignatovo Distr.: NPS, Aleksandrovskoe forestry, 20.V.2008, 1 ex.; 4 km S Barakhmany, 22.V.2008, 2 ex.; Ichalk Distr.: NPS, Lvovskoe forestry, 15 km NNE Ichalki, 7.VI.2017, 8.VI.2017, 7.VII.2017, 4 ex., Semishin G.B.; Ardatov Distr.: Probuzhdenie, 2.VII.2008, 1 ex.; Svetotekhnika, 18.V.2008, 2 ex.; Chamzinka Distr.: Komsomolskij, 28.V.2008, 1 ex., Ryzhov M.K.; Lyambir Distr.: Ekaterinovka, 29.V.2008, 2 ex.; 2.VI.2009, 1 ex.; Kadoshkino Distr.: Latyshovka, 4.VI.2016, 1 ex.; Insar Distr.: Vasina Polyana, 4.VI.2016, 1 ex.; Novlej, 27.V.2017, 1 ex.; Ruzaevka Distr. Yakovshchina, 26.VI.2016, 1 ex.; Kochkurovo Distr.: Starye Turdaki, 8.VII.2008, 1 ex.; Saransk, 20.VI.2009, 1 ex.

DISTRIBUTION in Mordovia. Temnikov Distr. [Ruchin, Kurmaeva, 2009; Feoktistov, 2011; Orlov et al., 2011; Egorov, Ruchin, 2012, 2013a, 2014; Egorov et al., 2015, 2016, 2017]; Atyurjevo Distr. [Ruchin, Kurmaeva, 2009]; Bolshoe Ignatovo Distr. [Ruchin, 2008a]; Ichalki Distr. [Ruchin, 2008a; Ruchin, Grishutkin, 2008; Semishin, 2009]; Ruzaevka Distr. [Ruchin, 2008a]; Lyambir Distr. [Semishin, 2009]; Kochkurovo Distr. [Semishin, 2009]. Saransk Town [Ruchin, 2009]. Elniki Distr., Krasnoslobodsk Distr, Ardatov Distr., Chamzinka Distr., Kadoshkino Distr., Insar Distr. [all this paper]
BIOLOGY in Mordovia. Occurs in mixed and deciduous forests, mature pine forests with spruce, birch, aspen, birch forests, alder forests, on floodplain and steppe meadows. The biotopes are meadows, glades, fringes, ruderal areas, sometimes overgrowth on the banks of water bodies. It is sometimes mown off with Urtica sp., Ranunculus sp., Inula sp. Imago flight lasts from the 2 nd decade of May to the 1 st decade of July.

\section{? Agapanthia violacea (Fabricius, 1775)}

DISTRIBUTION in Mordovia. Ichalki Distr. [Ruchin, 2008a]. Bolshie Berezniki Distr. [Timraleev, 1999].

BIOLOGY in Mordovia. Larvae develop in the stems of various herbaceous plants [Shapovalov, 2012].

\section{? Agapanthiola leucaspis (Steven, 1817)}

DISTRIBUTION in Mordovia. Temnikov Distr. [Plavilshchikov, 1964].

REMARK. The only indication of the species refers to the collections of S.M. Nismerchuk in the 1940s.

Thus, currently on the territory of Mordovia Republic, have been reliably detected 103 species of Cerambycidae. Three more species require confirmation with the collection material (Chlorophorus varius, Agapanthia violacea and Agapanthiola leucaspis), as they are known only from literature data. Habitation of Rhagium bifasciatum, Brachyta variabilis, Stenurella jaegeri, within the region is on the whole doubtful, so they are excluded from the list of fauna.

ACKNOWLEDGEMENTS. The authors are sincerely grateful to M.L. Danilevsky (Institute of Ecology and Evolution of the Russian Academy of Sciences, Moscow) for help in identifying certain species and valuable advice on the preparation of the manuscript; G.B. Semishin, D.K. Kurmaeva, S.V. Susarev, E.A. Lobachev, A.A. Benediktov, T. Malygina, O.N. Artayev and M.K. Ryzhov (Saransk), L.V. Bolshakov (Tula) for kindly provided material for processing.

\section{References}

Adlbauer K. 2001. 2. Nachtrag zur Bockkäferfauna der Steiermark unter dem Aspekt der Artenbedrohung (Coleoptera, Cerambycidae) // Joannea Zool. Vol.3. P.83-104.

Aleksanov V.V., Alekseev S.K. 2003. [A preliminary checklist of (Coleoptera, Cerambycidae) of the State Nature Reserve "Kaluzhskie Zaseki" and adjacent territories] // Arkhivy gosudarstvennogo prirodnogo zapovednika "Kaluzhskiye zaseki" Vol.1. Kaluga: Poligraf-Inform. P.111-115 [in Russian].

Bartenev A.F. 2004. [A review of the long-horned beetles species (Coleoptera: Cerambycidae) of the fauna of Ukraine] // Byulleten Kharkovskogo entomologicheskogo obshchestva. Vol.9. Nos1-2. P.24-43 [in Russian].

Bolshakov L.V.; Dorofeev Yu.V. 2004. [Coleoptera family Cerambycidae, or longicorns (Hexapoda: Coleoptera: Cerambycidae) of Tula region] // Biologicheskoye raznoobraziye Tul'skogo kraya na rubezhe vekov. Tula: Grif and $\mathrm{K}^{\circ}$. No.4. P.8-30 [in Russian]

Bondarenko N.V. 1964. [Species composition of fauna of insects and reproduction of forest pests of Mordovia reserve in 1948] // Trudy Mordovskogo gosudarstvennogo prirodnogo zapovednika. No.2. P.81-103 [in Russian].

Catalogue of Palaearctic Coleoptera. Vol. 6. Chrysomeloidea // I. Löbl, A. Smetana 2010. (eds.). Stenstrup: Apollo Books. 924 p. 
Cherepanov A.I. 1981. [Longicorn beetles of North Asia (Cerambycinae).] Novosibirsk: Nauka. 216 p. [In Russian].

[Coleopterous insects (Insecta, Coleoptera) of Republic of Adygheya (annotated catalogue of species) (Fauna conspecta of Adygheya. No.1)] // A.S. Zamotajlov, N.B. Nikitsky 2010. (eds.). Maykop: Adyghei State University Publishers. 404 pp [In Russian]

Danilevsky M.L. 2005. A checklist of the longicorn-beetles (Coleoptera: Cerambycidae) of Moscow region // Rus. Entomol. J. Vol.15. No.1. P.43-51.

Danilevsky M.L. 2014. [Longicorn beetles (Coleoptera, Cerambycoidea) of Russia and adjacent countries.] Part 1. Moscow: HSC. 518 p. [In Russian]

Danilevsky M.L. 2017. A check list of the longicorn beetles (Cerambycoidea) of Russia // https://www.zin.ru/Animalia/Coleoptera/ rus/dbase $31 . h t m$

Danilevskiy M.L., Miroshnikov A.I. 1985. [Longicorn beetles of Caucasus (Coleoptera, Cerambycidae).] Krasnodar. 419 p. [In Russian].

Dascălu M.M., Serafim R., Lindelov A. 2013. Range expansion of Trichoferus campestris (Faldermann) (Coleoptera: Cerambycidae) in Europe with the confirmation of its presence in Romania // Entomologica Fennica. Vol.24. P.142-146.

Dedyukhin S.V. 2007. [New data on the Udmurt fauna and spreading of Longicorn Beetles (Coleoptera: Cerambycidae)] // Vestnik Udmurtskogo universiteta. Biologiya. No.10. P.65-69 [in Russian].

Doychev D., Bencheva S. 2008. First record of Callidium coriaceum Paykull (Coleoptera: Cerambycidae) in Bulgaria // Silva Balcanica. Vol.9. No.1. P.97-99.

Egorov L.V. 2005. [Longicorn Beetles (Coleoptera: Cerambycidae) of the Chuvash Republic: a Taxonomic List of the Species] // Eversmannia. No.2. P.9-23 [in Russian].

Egorov L.V. 2006. [Addition to the fauna of longicorns (Coleoptera: Cerambycidae) of Chuvashia] // Eversmannia. No.7-8. P.25-26 [in Russian].

Egorov L.V. 2009. [Beetles (Insecta, Coleoptera) of Prisursky state nature reserve and its protected zone: the current state of knowledge] // Nauchnyye trudy gosudarstvennogo prirodnogo zapovednika "Prisurskiy", Vol.22. P.12-33 [in Russian].

Egorov L.V., Ruchin A.B. 2012. [Some data concerning the Coleoptera fauna of Mordovia State Nature Reserve] // Trudy Mordovskogo gosudarstvennogo prirodnogo zapovednika. Vol.10. P.4-57 [in Russian]

Egorov L.V., Ruchin A.B. 2013a. [Some data concerning the Coleoptera fauna of the Mordovia State Nature Reserve. Information 2] // Trudy Mordovskogo gosudarstvennogo prirodnogo zapovednika. Vol.11. P.133-192 [in Russian].

Egorov L.V., Ruchin A.B. 2013b. [New data on rare species of insects (Arthropoda, Insecta-Ectognatha) of Mordovia] // Vestnik Mordovskogo universiteta. No.3-4. P.116-121 [in Russian].

Egorov L.V., Ruchin A.B. 2014. [Some data concerning the Coleoptera fauna of the Mordovia State Nature Reserve. Information 3] // Trudy Mordovskogo gosudarstvennogo prirodnogo zapovednika. Vol.12. P.26-78 [in Russian].

Egorov L.V., Ruchin A.B., Alekseev S.K. 2010. [Addition to the coleoptera fauna (Insecta, Coleoptera) of the Mordovia state nature reserve] // Nauchnyye trudy gosudarstvennogo prirodnogo zapovednika "Prisurskiy". Vol.24. P.45-49 [in Russian]

Egorov L.V., Ruchin A.B., Semishin G.B. 2015. [Some data concerning the Coleoptera fauna of the Mordovia State Nature Reserve. Information 4] // Trudy Mordovskogo gosudarstvennogo prirodnogo zapovednika. Vol.14. P.82-156 [in Russian].

Egorov L.V., Ruchin A.B., Semishin G.B. 2016. [Some data concerning the Coleoptera fauna of Mordovia State Nature Reserve. Information 5] // Trudy Mordovskogo gosudarstvennogo prirodnogo zapovednika. Vol.16. P.293-364 [in Russian].

Egorov L.V., Ruchin A.B., Semishin G.B. 2017. [Some data concerning the Coleoptera fauna of the Mordovia State Nature Reserve. Information 6] // Trudy Mordovskogo gosudarstvennogo prirodnogo zapovednika. Vol.18. P.81-143 [in Russian]

Egorov L.V., Shapovalov A.M. 2017. On the distribution of a poorly known longicorn beetle, Phymatodes abietinus Plavilstshikov et
Lurie, 1960 (Coleoptera, Cerambycidae: Cerambycinae) // Entomological Review. Vol.97. No.3. P.353-356.

Fasulati K.K. 1971. [Field studying of land invertebrates.] 2 ed. Moscow: Vysshaya Shkola. 424 p. [In Russian]

Feoktistov V.F. 2011. [The list of insect species discovered for the first time in the Mordovia State Nature Reserve and in adjacent territories] // Vestnik Mordovskogo universiteta. Vol.4. P.8389 [in Russian].

Foit J. 2010. False click beetles (Eucnemidae) and longhorn beetles (Cerambycidae) of Dlouholoučské Stráně Nature Reserve (Eastern Bohemia) // Vč. sb. př́r. Práce a studie. Vol.17. P.187-194.

Georgiev G., Gjonov I., Sakalian V. 2015. New records of longhorn beetles (Coleoptera: Cerambycidae) in Strandzha Mountain // J. Entomol. Res. Soc. Vol.17. No.2. P.73-88.

Grebennikov V.V., Gill B.D., Vigneault R. 2010. Trichoferus campestris (Falmermann) (Coleoptera: Cerambycidae), an Asian woodboring beetle recorded in North America // Coleopterists Bulletin. Vol.64. P.13-20. doi: 10.1649/0010-065X-64.1.1

Hidalgo-Fontiveros A. 2010. Nuevos datos sobre cerambícidos de la provincia de Jaén (Andalucía, España) (Coleoptera, Cerambycidae) // Revista gaditana de Entomología. Vol.1. No.1. P.14-23 [In Español].

Isaev A.Yu. 2007. [The determinant of the coleopterans of the Middle Volga region (Part III: Polyphaga-Phytophaga)]. Ulyanovsk: Publisher Vector-S. 256 p. [in Russian].

Isaev A.Yu., Egorov L.V., Egorov K.A. 2004. [Beetles (Insecta, Coleoptera) of forest-steppe zone of the Middle Volga. Catalog.] Ulyanovsk: USU. 72 p. [In Russian]

Isaev A.Yu., Ishutov A.L. 2001. [Addition to the fauna of longicorn beetles (Coleoptera, Cerambycidae) of the Ulyanovsk region] // Nauchnyye trudy Gosudarstvennogo Prirodnogo Zapovednika "Prisurskiy". Vol.7. P.86-91 [in Russian].

Isaev A.Yu., Magdeev D.V. 2003. [Fauna of beetles-beetles (Coleoptera, Cerambycidae) of the forest-steppe zone of the Volga region] // Issledovaniya v oblasti biologii i metodiki yeye prepodavaniya: mezhvuzovskiy sbornik nauchnykh trudov $\mathrm{k} 100$ letiyu D.N. Florova. No.3. Part 1. Samara. P.290-300 [in Russian].

Kadyrov A.Kh., Karpiński L., Szczepański W.T., Taszakowski A., Walczak M. 2016. New data on distribution, biology, and ecology of longhorn beetles from the area of west Tajikistan (Coleoptera, Cerambycidae) // Zookeys. Vol.606. P.41-64. doi: 10.3897/zookeys.606.9190.

Kirsta L.V. 1974. [Distribution and role of pine beetle drivers in the stands of the Mordovia reserve] // Trudy Mordovskogo gosudarstvennogo prirodnogo zapovednika No.6. P.134-140 [in Russian].

Kurzawa J. 2012. Distribution of Rhaphuma gracilipes (Faldermann, 1835) (Coleoptera: Cerambycidae) in Europe // Acta entomologica silesiana. Vol.20. P.65-70.

Mozolevskaya A.G., Chekanov M.I., Chekanova O.P. 1971. [Dendrophilous insects Mordovia reserve] // Trudy Mordovskogo gosudarstvennogo prirodnogo zapovednika No.5. P.199-218 [in Russian].

Navarro J., Urbano J.M., Llinares A. 2005. Deilus fugax (Olivier, 1790) (Coleoptera, Cerambycidae) especie presente en Sevilla (Andalucía, España) // Boletín de la Sociedad Andaluza de Entomología. No.12. P.51-56.

Orlov A.A., Ruchin A.B., Khapugin A.A. 2011. [About anthophilic beetles in P.G. Smidovich Mordovia state nature reserve] // Vestnik Mordovskogo universiteta. No.4. P.194-197 [in Russian]

Pennacchio F., Marianelli L., Binazzi F., Francardi V., Paoli F., Griffo R., Roversi P.F. 2016. First interception of Trichoferus campestris (Faldermann, 1835) (Coleoptera Cerambycidae Cerambycinae) in Italy// REDIA. Vol.99. P.59-62. http://dx.doi.org/ 10.19263/REDIA-99.16.06.

Plavilshchikov N.N. 1964. [A list of insect species found on the territory of the Mordovia State Nature Reserve] // Trudy Mordovskogo gosudarstvennogo prirodnogo zapovednika. Vol.2. P.105-134 [in Russian].

Plewa R., Łoś K., Górski P. 2011. New data on the distribution, biology and behavior of some longhorn beetles (Coleoptera, 
Cerambycidae) from Greece // Elateridarium. Vol.5. P.232-247 [in Polish]

[Red Data Book of the Republic of Mordovia. 2005. Vol.2. Animals.] Saransk: Publisher of the Mordovia State University. 336 p. [In Russian]

Redikortsev V.V. 1938. [Materials to the entomofauna of the Mordovia State Nature Reserve] // Fauna Mordovskogo gosudarstvennogo zapovednika imeni P.G. Smidovich. Moscow: Committee of Nature Reserves at the Presidium of the All-Union Central Executive Committee. P.137-146 [in Russian].

Ruchin A.B. 2008a. [Materials on the fauna of longicorn beetles (Coleoptera: Cerambycidae) of Mordovia] // Vestnik Mordovskogo universiteta. No.2. P.51-58 [in Russian].

Ruchin A.B. 2008b. [New species of beetles (Coleoptera, Insecta) of the Republic of Mordovia] // Organisms, populations, ecosystems: problems and ways of biodiversity conservation. Vologda. P.237-239 [in Russian].

Ruchin A.B. 2009. [Species composition and some aspects of logicorns biology (Coleoptera, Cerambycidae) in Saransk] // Vestnik Mordovskogo universiteta. No.1. P.57-58 [in Russian].

Ruchin A.B. 2011. [First additional materials towards the entomofauna of the Mordovia State Nature Reserve] // Trudy Mordovskogo gosudarstvennogo prirodnogo zapovednika. Vol.9. P.150-182 [in Russian].

Ruchin A.B. 2015a. [Second additional materials on the insect fauna of the Mordovia State Nature Reserve] // Trudy Mordovskogo gosudarstvennogo prirodnogo zapovednika. Vol.13. P.351-398 [in Russian].

Ruchin A.B. 2015b. [Xylophagous insects of Mordovia reserve] // Mordovia reserve. No.8. P.6-7 [in Russian].

Ruchin A.B., Egorov L.V. 2013. [New data on the coleopteran fauna of the NP Smolny (Republic of Mordovia)] // Izvestiya vysshikh uchebnykh zavedeniy. Povolzhskiy region. Yestestvennyye nauki. No.3. P.21-28 [in Russian].

Ruchin A.B., Egorov L.V. 2015. [Coleoptera species, recommended for protection (Coleoptera) in the Republic of Mordovia (main list of protected taxa)] // Trudy Mordovskogo gosudarstvennogo prirodnogo zapovednika imeni P.G. Smidovicha. No.15. P.70104 [in Russian].

Ruchin A.B., Egorov L.V. 2018. Leptura aurulenta (Coleoptera, Cerambycidae), a new record of a very rare species in Russia // Nature Conservation Research. Vol.3. No.1. P.88-91. DOI: 10.24189/ncr.2018.003

Ruchin A.B., Egorov L.V., Alekseev S.K., Chikhlyaev I.V., Fayzulin A.I. 2015. [Data on findings of rare species of invertebrates and vertebrates in the Republic of Mordovia in 2013-2014] // Trudy Mordovskogo gosudarstvennogo prirodnogo zapovednika. Vol.14. P.333-344 [in Russian].

Ruchin A.B., Egorov L.V., Alekseev S.K., Kurmaeva D.K., Ryzhov M.K., Semishin G.B. 2009. [New species of coleoptera (Insecta: Coleoptera) in fauna of Mordovia Republic] // Izvestiya Kaluzhskogo obshchestva izucheniya prirody. Book 9. Kaluga. Publishing house of K.E. Tsiolkovsky KSPU. P.73-86 [in Russian].

Ruchin A.B., Egorov L.V., Alekseev S.K., Lobachev E.A., Kurmaeva D.K. 2010. [Materials on new findings of rare species of coleoptera (Insecta, Coleoptera) in fauna of Mordovia Republic] // Aktualnyye voprosy sovremennoy entomologii i ekologii nasekomykh. Borisoglebsk. P.58-61 [in Russian].

Ruchin A.B., Egorov L.V., Artaev O.N., Alekseev S.K., Zavjalov N.A. 2014. [New data on rare species of invertebrates and vertebrates in the Republic of Mordovia with discussion of protection status of some species] // Trudy Mordovskogo gosudarstvennogo prirodnogo zapovednika. Vol.12. P.196-216 [in Russian].

Ruchin A.B., Egorov L.V., Artaev O.N., Nikolaeva A.M. 2016. [New data on rare species of invertebrates and vertebrates in the Republic of Mordovia] // Trudy Mordovskogo gosudarstvennogo prirodnogo zapovednika. Vol.16. P.405-418 [in Russian].

Ruchin A.B., Egorov L.V., Nikolaeva A.M., Mikhailenko A.P 2017. [New data on rare species of invertebrates in Mordovia]. Molodoy Uchenyi. Vol.2. No.136. P.234-240 [in Russian].

Ruchin A.B., Grishutkin G.F. 2008. [First information about longicorn beetles fauna (Coleoptera: Cerambycidae) of the Smolny National Park] // Biologicheskaya nauka i obrazovaniye v pedagogicheskikh vuzakh. No.5. Novosibirsk. P.68-69 [in Russian]

Ruchin A.B., Kurmaeva D.K. 2009. [Materials on longicorns fauna (Coleoptera: Cerambycidae) of Mordovia Reserve and adjacent territories] // Samarskaya Luka: problemy regionalnoy i globalnoy ekologii. Vol.18. No.2. P.129-134 [in Russian].

Ruchin A.B., Kurmaeva D.K. 2010. On rare insects of Mordovia included in the Red Data Book of the Russian Federation. Entomol Review. Vol.90. No.6. P.712-717.

Ruchin A.B., Loginova N.G., Kurmaeva D.K. 2007. [A contribution to the insect fauna of two forestry areas of the National Rark "Smolny"] // Fauna i ecologiya nasekhomyh. Vol.1. Rostov-onDon: Tsentry Valentnosti Vuzov Rossii Publishing House. P.2433 [in Russian].

Ryzhov M.K. 2012. [Finds of rare and new species of animals and plants in Mordovia in 2011] // Trudy Mordovskogo gosudarstvennogo prirodnogo zapovednika imeni P.G. Smidovicha. No.10. P.357-358 [in Russian].

Semishin G.B. 2009. [Some materials on the finds of beetles (Coleoptera) in Mordovia] // Vestnik Mordovskogo universiteta. No.1. P.253-255 [in Russian].

Shapovalov A.M. 2012. [Longicorns (Coleoptera, Cerambycidae) of Orenburg region: fauna, distribution, bionomics] // Trudy Orenburgskogo otdeleniya Russkogo entomologicheskogo obshchestva. No.3. 221 p. [In Russian]

Sláma M., Gutowski J.M. 1997. Xylotrechus capricornis (Gebler, 1830) (Coleoptera: Cerambycidae) - new to the Polish and Czech fauna // Wiad. entomol. Vol.16. No.2. P.83-97 [in Polish].

Timraleev Z.A. 1999. [Fauna and ecology of the longicorns in Mordovian Prisurie] // Nauchnyye trudy Gosudarstvennogo Prirodnogo Zapovednika "Prisurskiy". Vol.2. P.43-45 [in Russian].

Timraleev Z.A., Kamenev A.G., Bardin O.D. 2007. [Insects of Mordovia. Part II. Coleoptera.] Saransk: Mordovia University Publishing House. 176 p. [In Russian]

Vlasov D.V. 1999. [Fauna of longicorns in Yaroslavl region: the degree of knowledge and perspectives of studying] // Biologicheskiye resursy, ikh sostoyaniye $\mathrm{i}$ ispol'zovaniye $\mathrm{v}$ basseyne Verkhney Volgi. Yaroslavl. P.220-229 [in Russian].

Vlasov D.V. 2015. [Longicorn beetles (Coleoptera, Cerambycidae) of Pleshcheevo Lake National Park (Yaroslavl region)] // Nauchnyye trudy gosudarstvennogo prirodnogo zapovednika "Prisurskiy". Vol.30. No.1. P.73-78 [in Russian].

Yamashkin AA. 1998. [Physical and geographical conditions of the Republic Mordovia.] Saransk: Mordovia University Publishing House. 156 p. [in Russian] 